\title{
Dedekind sums for Hecke groups
}

by

Roelof W. Bruggeman (Utrecht)

\section{Introduction and statement of results}

1.1. Hecke groups. The Hecke groups $G(\lambda)$, with $\lambda>0$, are the discrete subgroups of $\mathrm{SL}_{2}(\mathbb{R})$ generated by $\left(\begin{array}{ll}1 & \lambda \\ 0 & 1\end{array}\right)$ and $\left(\begin{array}{cc}0 & 1 \\ -1 & 0\end{array}\right)$. The condition of discreteness implies that either $\lambda=2 \cos (\pi / q)$ with $q=3,4, \ldots$, or $\lambda \geq 2$. This is the class of discrete groups for which Hecke [14] considered the relation between Dirichlet series and automorphic forms; see also [15]. In [19] and [20], Maass extends Hecke's theory to real analytic automorphic forms.

This paper concerns the case $\lambda<2$ only, hence $\lambda=2 \cos (\pi / q)$. Notation: $G_{q}=G(2 \cos \pi / q)$. It is well known that $G_{3}=\mathrm{SL}_{2}(\mathbb{Z})$, the modular group. Conjugates of the Hecke groups $G_{4}$ and $G_{6}$ are commensurable to $G_{3}$. The other $G_{q}$ are incommensurable to conjugates of $G_{3}$ and of each other; see [18]. These $G_{q}$ are called non-arithmetical as subgroups of $\mathrm{SL}_{2}(\mathbb{R})$.

1.2. Dedekind sums. The classical Dedekind sum $S(d, c)$, with $c, d \in \mathbb{Z}$, $d>0, c$ and $d$ relatively prime, is a rational number that occurs in the transformation formula for the eta function of Dedekind; see R. Dedekind's Erläuterungen zu den Fragmenten XXVIII in Riemann's collected papers [10]; especially formula (12) on p. 469, or see [24], p. 47. Dedekind obtains an elementary expression for $S(d, c)$, see $(32)$ on p. 475 ; it is equivalent to

$$
\begin{aligned}
S(d, c) & =\sum_{x \bmod c}\left(\left(\frac{d x}{c}\right)\right)\left(\left(\frac{x}{c}\right)\right), \\
((u)) & = \begin{cases}u-1 / 2 & \text { if } 0<u<1, \\
0 & \text { if } u=0, \\
((u+n)) & \text { for all } n \in \mathbb{Z} .\end{cases}
\end{aligned}
$$

In [24], Rademacher uses this expression as the definition.

Goldstein has generalized Dedekind's approach to general cofinite discrete subgroups of $\mathrm{SL}_{2}(\mathbb{R})$; see [11]-[13]. This can be applied to the $G_{q}$ and gives a number $T(c, d) \in \mathbb{Q}(\lambda)$ for each $(c, d)$ that occurs as the lower row 
of an element of $G_{q}$, and satisfies

$$
\begin{aligned}
T(1,0) & =0, \\
T\left(c, d_{1}\right) & =T(c, d) \quad \text { if } d_{1}-d \in \lambda c \mathbb{Z}, \\
T(c,-d) & =-T(c, d), \\
T(-c,-d) & =T(c, d), \\
T(c, d)+T(d, c) & =(q-2) \frac{c^{2}+d^{2}+1}{\lambda c d}-q \quad \text { if } c, d>0 .
\end{aligned}
$$

(I do not follow Goldstein's normalization.) These relations determine $T(c, d)$ recursively. In the modular case $(q=3)$ the same relations are satisfied by 12 times the classical Dedekind sum; hence $T(c, d)=12 S(d, c)$ if $q=3$.

As far as I know, an elementary, non-recursive, expression for the $T(c, d)$ is available only in the arithmetical cases $q=3,4,6$.

1.3. Distribution of Dedekind sums. The Dedekind sums turn out to satisfy $T(d, c)=\mathcal{O}(c)(c \rightarrow \infty)$. So the $T(c, d) / c$ are elements of a bounded interval. We can get information concerning the distribution of $(a / c, d / c$, $T(c, d) / c$ ) where $\left(\begin{array}{ll}a & b \\ c & d\end{array}\right)$ runs through the elements of $G_{q}$ that satisfy $c>0$, $-\lambda c / 2<a \leq \lambda c / 2$ and $-\lambda c / 2<d \leq \lambda c / 2$. In the next propositions we denote by $\sum_{(c, d)}^{r}$ the sum over the lower rows that satisfy these restrictions. For a given pair $(c, d)$ there is exactly one matrix $\left(\begin{array}{ll}a & b \\ c & d\end{array}\right) \in G_{q}$ satisfying these conditions.

1.4. Proposition (First distribution result). For all continuous functions $f$ on $(\mathbb{R} \bmod \lambda \mathbb{Z})^{2} \times \mathbb{R}$

$$
\lim _{X \rightarrow \infty} \frac{1}{X^{2}} \sum_{(c, d), c<X}^{r} f\left(\frac{a}{c}, \frac{d}{c}, \frac{T(c, d)}{c}\right)=\frac{q}{\pi^{2}(q-2)} \int_{0}^{\lambda} \int_{0}^{\lambda} f(\xi, \eta, 0) d \xi d \eta .
$$

1.5. Proposition (Second distribution result). For each continuous function $g$ on $(\mathbb{R} \bmod \lambda \mathbb{Z})^{2} \times \mathbb{R}$ define the function $f$ by $f(\xi, \eta, \sigma)=$ $\sigma^{2} g(\xi, \eta, \sigma)$. Then

$$
\begin{aligned}
\lim _{X \rightarrow \infty} \frac{1}{X} \sum_{(c, d), c<X}^{r} f\left(\frac{a}{c},\right. & \left.\frac{d}{c}, \frac{T(c, d)}{c}\right) \\
& =\sum_{\left(c_{1}, d_{1}\right)}^{r} \sum_{\left(c_{2}, d_{2}\right)}^{r} \frac{1}{\lambda c_{1} c_{2}} \sum_{ \pm} f\left(\frac{a_{1}}{c_{1}}, \frac{d_{2}}{c_{2}}, \frac{ \pm(q-2)}{\lambda c_{1} c_{2}}\right) .
\end{aligned}
$$


Remarks. The first distribution result states that the majority of the $T(c, d) / c$ are concentrated near zero, and that the distribution is uniform with respect to $(a / c, d / c)$. The quadratic factor in the test functions that occur in the second distribution result masks this majority near zero. The minority staying away from 0 turns out to be concentrated near a set of limit points.

In [8], $\S 3$, I have stated these results for the modular case $q=3$. I have proved two-dimensional versions of these results (without the dependence on $a / c)$ in [5], for the modular case only.

The proof, in Sections 5-7, will go along the same lines as in [5]; it takes some more work to include the dependence on $a / c$. The proof uses the meromorphic continuation of Eisenstein and Poincaré series in spectral parameter and weight jointly. This continuation is given in [2], [3] for the modular case. The results we need can be obtained in exactly the same way for the $G_{q}$ as for $G_{3}$. Full proofs one finds in [9], in a much wider context.

These continuation results can be extended to $G(2)$, the theta group, but are more complicated, due to the presence of two cusps instead of one. I think that one can get distribution results for Dedekind sums associated with $G(2)$ as well, but they will be more complicated. For $G(\lambda)$ with $\lambda>2$ I do not know what would be the right concept of Dedekind sum. These discrete groups have a fundamental domain with infinite area.

1.6. Limit points. Let $G_{q}^{0}=\left\{\left(\begin{array}{ll}a & b \\ c & d\end{array}\right) \in G_{q}: c \neq 0\right\}$. The distribution results above give information concerning the image of the map

$$
\beta: G_{q}^{0} \rightarrow \mathbb{R}:\left(\begin{array}{ll}
a & b \\
c & d
\end{array}\right) \mapsto\left(\frac{a}{c}, \frac{d}{c}, \frac{T(c, d)}{c}\right) .
$$

Clearly

$$
\beta\left(\left(\begin{array}{ll}
1 & \lambda \\
0 & 1
\end{array}\right)\left(\begin{array}{ll}
a & b \\
c & d
\end{array}\right)\right)=\beta\left(\begin{array}{ll}
a & b \\
c & d
\end{array}\right)+(\lambda, 0,0)
$$

and

$$
\beta\left(\left(\begin{array}{ll}
a & b \\
c & d
\end{array}\right)\left(\begin{array}{ll}
1 & \lambda \\
0 & 1
\end{array}\right)\right)=\beta\left(\begin{array}{ll}
a & b \\
c & d
\end{array}\right)+(0, \lambda, 0) .
$$

This explains the use of periodic test functions.

Let $\mathcal{P}$ be the plane $\{(\xi, \eta, 0): \xi, \eta \in \mathbb{R}\}$ in $\mathbb{R}^{3}$, and let

$$
\mathcal{L}=\left\{\left(\frac{a_{1}}{c_{1}}, \frac{d_{2}}{c_{2}}, \pm \frac{q-2}{\lambda c_{1} c_{2}}\right):\left(\begin{array}{ll}
a_{j} & b_{j} \\
c_{j} & d_{j}
\end{array}\right) \in G_{q} \text { for } j=1,2\right\} .
$$

From the distribution results it follows that all points of $\mathcal{P} \cup \mathcal{L}$ are limit points of $\beta\left(G_{q}^{0}\right)$. In Proposition 1.9 we shall see that there are no more limit points. 
Figures 1 and 2 give illustrations for Proposition 1.5; they are discussed in Section 2. In these figures the elements of $\mathcal{L}$ seem to be approached by lines of points. The next result describes this feature.

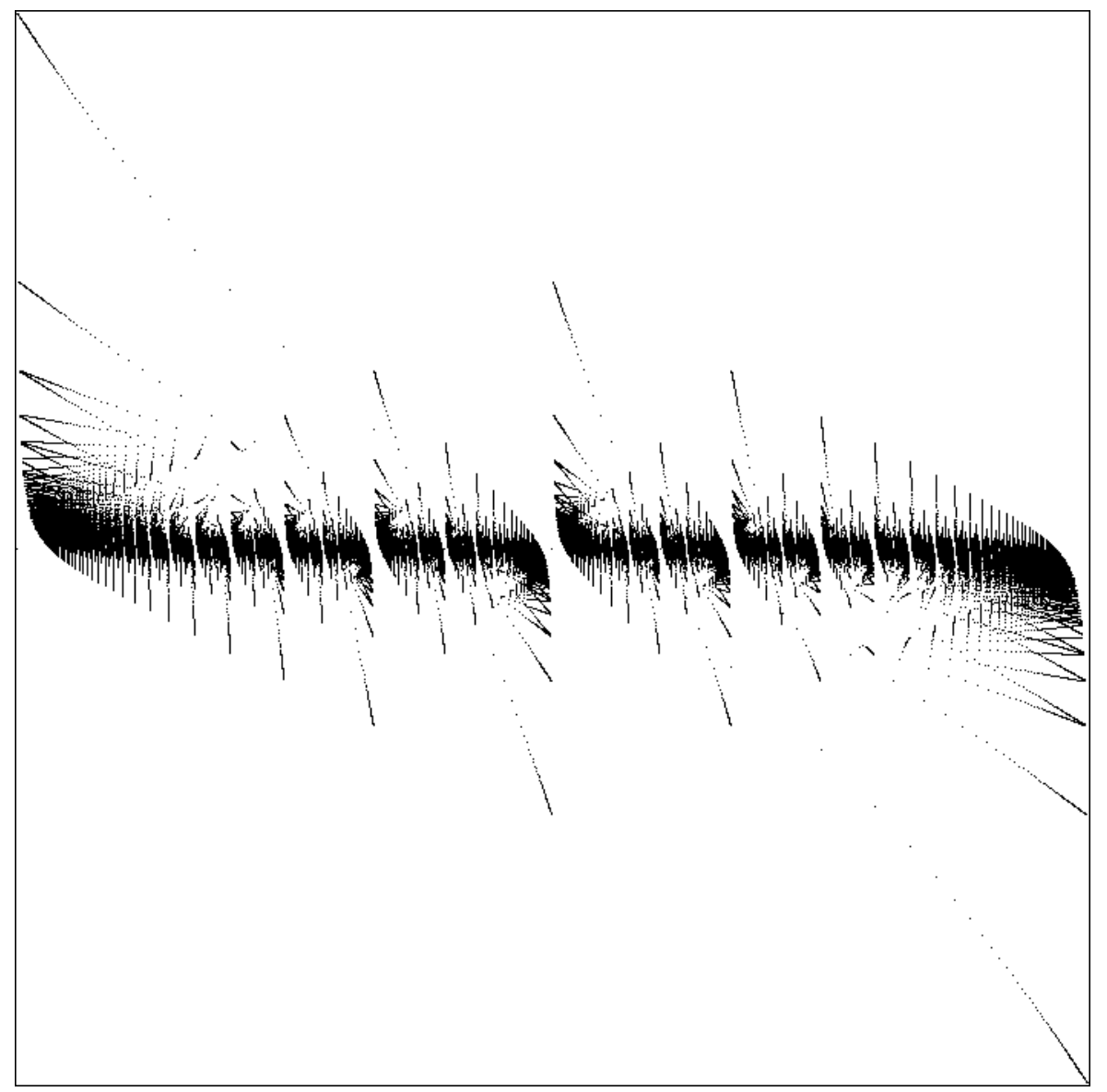

Fig. 1. $T(c, d) / c$ against $d / c$ for the modular group $G_{3}$, with $c<1000$. Number of points: 151897. Horizontal: $d / c \in[0.00,1.00)$, vertical: $T(c, d) / c \in[-1.00,1.00]$

1.7. Proposition. Let $\gamma_{j}=\left(\begin{array}{ll}a_{j} & b_{j} \\ c_{j} & d_{j}\end{array}\right) \in \Gamma$, with $c_{j}>0, j=1,2$. For $\pm r \rightarrow \infty, r \in \mathbb{Z}$

$$
\begin{aligned}
\beta\left( \pm \gamma_{1}\left(\begin{array}{cc}
1 & r \lambda \\
0 & 1
\end{array}\right) \gamma_{2}\right)=\left(\frac{a_{1}}{c_{1}}, \frac{d_{2}}{c_{2}}, \mp \frac{q-2}{\lambda c_{1} c_{2}}\right) \\
\quad+\frac{1}{\lambda r}\left(\frac{-1}{c_{1}^{2}}, \frac{-1}{c_{2}^{2}}, \frac{ \pm T\left(c_{1}, d_{1}\right) \pm T\left(c_{2}, d_{2}\right)+q}{c_{1} c_{2}}\right)+\mathcal{O}\left(\frac{1}{r^{2}}\right) .
\end{aligned}
$$

Pr o of. For the first and second coordinate a computation suffices. For the last coordinate use (3.1). 
Remarks. Elements of $\beta\left(G_{q}^{0}\right)$ approach the points in $\mathcal{L}$ along approximately straight lines. The proposition does not say that there are no other elements of $\beta\left(G_{q}^{0}\right)$ near points of $\mathcal{L}$.

D. Zagier pointed out this result for the modular case. He also suggested to look whether $\beta\left(G_{q}^{0}\right)$ is discrete outside $\mathcal{L} \cup \mathcal{P}$. Proposition 1.5 gives a density result only.

1.8. Notations. Let

$p=\left(a_{1} / c_{1}, d_{2} / c_{2}, \mp(q-2) / \lambda c_{1} c_{2}\right) \in \mathcal{L}, \quad$ with $\gamma_{j}=\left(\begin{array}{ll}a_{j} & b_{j} \\ c_{j} & d_{j}\end{array}\right) \in G_{q}, c_{j}>0$.

For $X>0$, put

$$
A^{ \pm}(p, X)=\left\{\beta\left(\gamma_{1}\left(\begin{array}{cc}
1 & r \lambda \\
0 & 1
\end{array}\right) \gamma_{2}\right): r \in \mathbb{Z}, \pm c\left(\gamma_{1}\left(\begin{array}{cc}
1 & r \lambda \\
0 & 1
\end{array}\right) \gamma_{2}\right)>X\right\}
$$

with $c(M)$ the left lower entry of the matrix $M$. Each $A^{ \pm}(p, X)$ is an end part of a "line" of points in $\beta\left(G_{q}^{0}\right)$ approaching $p$. The freedom in the choice of the $\gamma_{j}$ can be absorbed in a translation of $r$.

For any $p=\left(x_{1}, x_{2}, x_{3}\right) \in \mathbb{R}^{3}$ we define the $\varepsilon$-neighborhood $U_{\varepsilon}(p)$ by

$$
U_{\varepsilon}(p)=\left\{\left(y_{1}, y_{2}, y_{3}\right):\left|x_{j}-y_{j}\right|<\varepsilon \text { for } j=1,2,3\right\} .
$$

1.9. Proposition. For each $p=(\xi, \eta, \sigma) \in \mathbb{R}^{3}$ with $\sigma \neq 0$, there are $\varepsilon>0$ and $X>1$ such that

$$
\left\{\beta\left(\begin{array}{ll}
a & b \\
c & d
\end{array}\right) \in U_{\varepsilon}(p):\left(\begin{array}{ll}
a & b \\
c & d
\end{array}\right) \in G_{q}^{0}, c>X\right\}= \begin{cases}A^{ \pm}(p, X) & \text { if } p \in \mathcal{L} \\
\emptyset & \text { otherwise }\end{cases}
$$

Proof. See Section 4.

R e mark. In [6] I have discussed the results of Propositions 1.7 and 1.9 for the modular case, without the $a / c$-coordinate. The proofs are based on the recursive relations defining the Dedekind sums.

1.10. R e m a r k s. Clearly the $T(c, d)$ generalize the $12 S(d, c)$ of the modular case. I am not fully happy with the name (generalized) Dedekind sum. After all, a nice expression defining $T(c, d)$ directly as a sum depending on $c$ and $d$ does not exist in the non-arithmetic cases, as far as I know.

If one looks at the distribution results in the modular case, it seems that the set of limit points has a very arithmetical structure. It has surprised me that these results can be carried over to all Hecke groups $G_{q}$.

In [21], and in Proposition 13.6.2 of [9], the method of this paper leads to distribution results for other quantities.

One finds other distribution results for the classical Dedekind sums $(q=$ $3)$ in [26] and [7]. The methods are quite different from those in this paper. Extension of the results in [7] to the distribution of $(a / c, d / c, S(d, c))$, and 


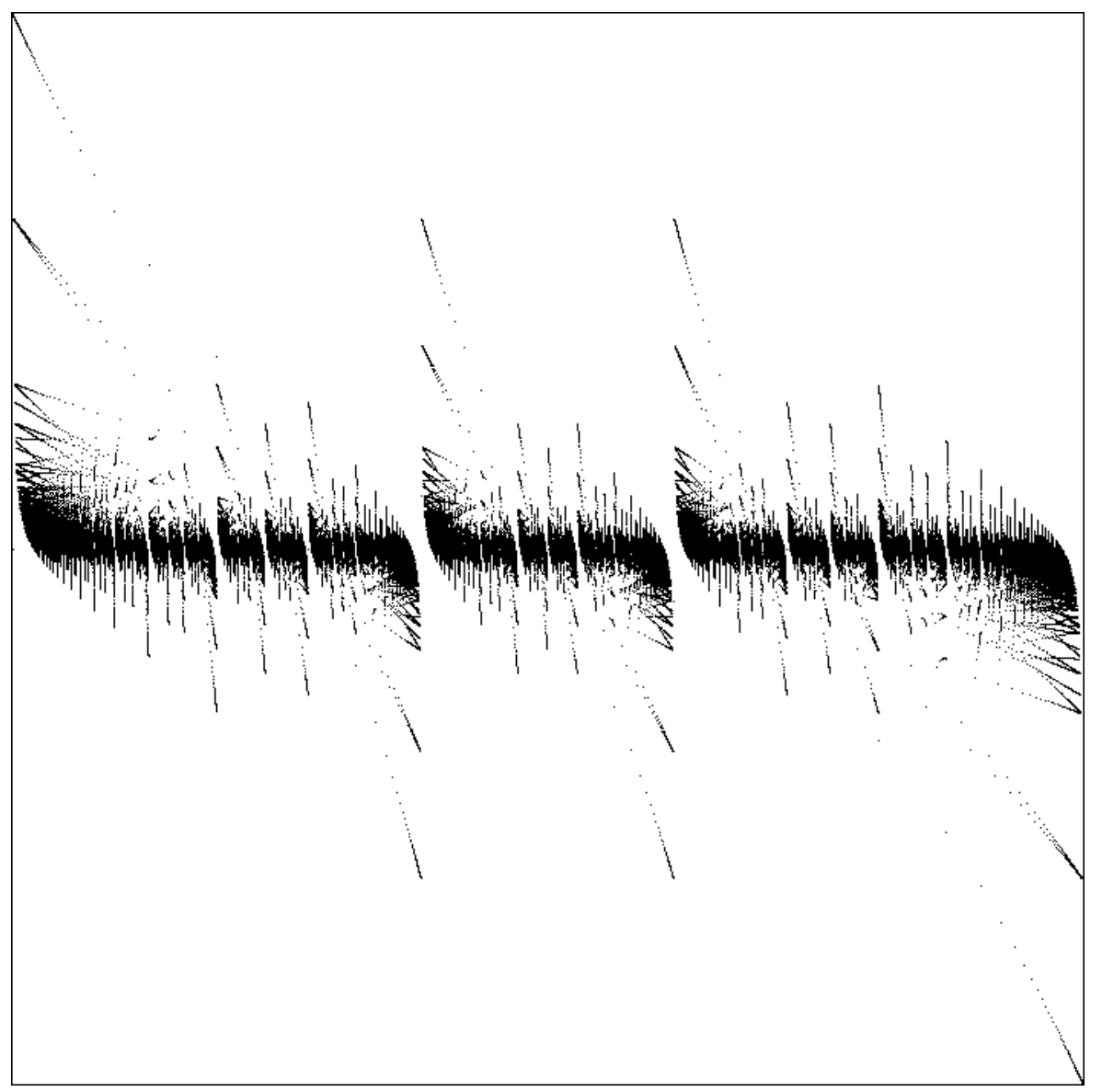

Fig. 2. $T(c, d) / c$ against $d / c$ for the Hecke group $G_{5}$, with $c<1000$. Number of points: 221080. Horizontal: $d / c \in[0.00,1.62)$, vertical: $T(c, d) / c \in[-1.85,1.85]$

to the Hecke groups $G_{q}$ might be possible. That extension is not the aim of this paper.

2. Figures. The distribution results ask to be illustrated. In this section, we look mainly at the case $q=5$.

It is difficult to get the three-dimensional structure in a picture. Here we are content to consider the distribution of $(d / c, T(c, d) / c)$. In Figures 1 and 2 we have given for $q=3$ and $q=5$ a plot of $(d / c, T(c, d) / c)$ with $(d, c)$ occurring as the lower row of an element in $G_{q}$, with the additional conditions $1 \leq c<1000,-\lambda c / 2<d \leq \lambda c / 2$.

In both figures we see limit points being approached along lines. The horizontal black strip is the majority of points with $T(c, d) / c$ approximately zero. We shall see in 2.4 that the gaps are due to the truncation $c<1000$. 
The figures suggest a selfsimilarity of the distribution. This is explained by a partial action of $G_{q} /\{ \pm I\}$ in $\mathbb{R}^{3}$ that leaves $\beta\left(G_{q}^{0}\right)$ approximately invariant; see 4.6 .

2.1. Figure 2. We take a closer look at Figure 2. The matrix elements of $G_{5}$ are in $\mathbb{Z}+\mathbb{Z} \lambda$, with $\lambda=\frac{1}{2}(1+\sqrt{5})$. The seven positive values of $c$ smaller than 6.0 are $1, \lambda \approx 1.62,2 \lambda \approx 3.24, \lambda+2 \approx 3.62,2 \lambda+1 \approx 4.24$, $3 \lambda \approx 4.85$, and $2+2 \lambda \approx 5.24$. For the corresponding matrices in $G_{5}$ that occur in the distribution results we have listed the values of $\Phi$ and $T$ in Table 1.

Table 1. Values of $\Phi$ and $T$ for the first few $(c, d)$ for $G_{5}$

\begin{tabular}{|c|c|c|}
\hline$(1,0)$ & $\Phi\left(\begin{array}{cc}0 & -1 \\
1 & 0\end{array}\right)=0$ & $T(1,0)=0$ \\
\hline$(\lambda, \pm 1)$ & $\Phi\left(\begin{array}{cc} \pm 1 & 0 \\
\lambda & \pm 1\end{array}\right)= \pm 2$ & $T(\lambda, 1)=10-6 \lambda$ \\
\hline$(2 \lambda, \pm 1)$ & $\Phi\left(\begin{array}{cc} \pm 1 & 0 \\
2 \lambda & \pm 1\end{array}\right)=\mp 1$ & $T(2 \lambda, 1)=7-3 \lambda$ \\
\hline$(\lambda+2, \pm \lambda)$ & $\Phi\left(\begin{array}{cc}\mp \lambda & -1 \\
\lambda+2 & \pm \lambda\end{array}\right)=0$ & $T(\lambda+2, \lambda)=0$ \\
\hline$(2 \lambda+1, \pm \lambda)$ & $\Phi\left(\begin{array}{cc} \pm 2 \lambda & 1 \\
2 \lambda+1 & \pm \lambda\end{array}\right)= \pm 1$ & $T(2 \lambda+1, \lambda)=18 \lambda-28$ \\
\hline$(2 \lambda+1, \pm 2 \lambda)$ & $\Phi\left(\begin{array}{cc} \pm \lambda & 1 \\
2 \lambda+1 & \pm 2 \lambda\end{array}\right)= \pm 1$ & $T(2 \lambda+1,2 \lambda)=18 \lambda-28$ \\
\hline$(3 \lambda, \pm 1)$ & $\Phi\left(\begin{array}{cc} \pm 1 & 0 \\
3 \lambda & \pm 1\end{array}\right)=\mp 4$ & $T(3 \lambda, 1)=8-2 \lambda$ \\
\hline$(2+2 \lambda, 2 \lambda+1)$ & $\Phi\left(\begin{array}{cc}2 \lambda+1 & 2 \lambda \\
2+2 \lambda & 2 \lambda+1\end{array}\right)=3$ & $T(2+2 \lambda, 2 \lambda+1)=0$ \\
\hline
\end{tabular}

2.2. Limit points. We consider a few limit points $\left(a_{1} / c_{1}, 0,3 / \lambda c_{1}\right)$ that are projected to $\left(0,3 / \lambda c_{1}\right)$ on the left hand side of Figure 2. (So $\gamma_{1}=\left(\begin{array}{l}a_{1} \cdot \\ c_{1}\end{array}\right.$.) and $\gamma_{2}=\left(\begin{array}{cc}0 & -1 \\ 1 & 0\end{array}\right)$.) The first four values of $c_{1}$ give $3 / \lambda \approx 1.85,3 / \lambda^{2} \approx 1.15$, $3 /\left(2 \lambda^{2}\right) \approx 0.57$, and $3 /(\lambda(\lambda+2)) \approx 0.51$. Proposition 1.7 implies that $\left(0,3 /\left(\lambda c_{1}\right)\right)$ is approximated by lines with direction $\left(1, \frac{T\left(c_{1}, d_{1}\right)-5}{c_{1}}\right)$.

The possibilities for $d_{1}$ are listed in Table 2. Note that for $c_{1}=\lambda+2$ both values of $d_{1}$ give the same direction.

Table 2. Direction of approach to some limit points for $G_{5}$ (compare Figure 2)

\begin{tabular}{|c|r|l|}
\hline$c_{1}$ & $d_{1}$ & Direction \\
\hline 1 & 0 & $(1,-5)$ \\
\hline$\lambda$ & 1 & $\begin{array}{l}(1,5 \lambda-11) \approx(1.00,-2.91) \\
(1,21-15 \lambda) \approx(1.00,-3.27)\end{array}$ \\
& -1 & $\begin{array}{l}(1, \lambda-5 / 2) \approx(1.00,-.88) \\
(1,15 / 2-6 \lambda) \approx(1.00,-2.21)\end{array}$ \\
\hline $2 \lambda$ & 1 & $(1, \lambda-3) \approx(1.00,-1.38)$ \\
\hline$\lambda+2$ & \pm 1 & \\
\hline
\end{tabular}


2.3. Selfsimilarity. The projection $p_{23} \beta\left(G_{5}^{0}\right)$ of $\beta\left(G_{5}^{0}\right)$ onto the $(\eta, \sigma)$ plane is invariant under $T_{1}:(\eta, \sigma) \mapsto(\eta+\lambda, \sigma)$, and $j:(\eta, \sigma) \mapsto(-\eta,-\sigma)$. In Figure 2 we have the union of two fundamental domains for the group generated by $T_{1}$ and $j$. In 4.6 we shall see that $p_{23} \beta\left(G_{5}^{0}\right)$ is also approximately invariant under $W:(\eta, \sigma) \mapsto(-1 / \eta, \sigma /|\eta|)$, defined on $\{(\eta, \sigma): \eta \neq 0\}$.

Consider, for instance, $(\eta, \sigma)=(\lambda-1+\delta, \sigma)$ with $\delta$ small. This means that $(\eta, \sigma)$ is in the vertical strip near the large gap left from the center in Figure 2 .

The composition $T_{1} \circ W$ sends $(\eta, \sigma)$ to

$$
\left(\frac{-1}{\eta}+\lambda, \frac{\sigma}{|\eta|}\right) \approx\left(\lambda^{2} \delta+\mathcal{O}\left(\delta^{2}\right), \lambda \sigma+\mathcal{O}(\delta)\right) .
$$

So the vertical strip near $\eta=\lambda-1$ is mapped to the vertical strip near $\eta=0$. (Imagine Figure 2 horizontally extended with period $\lambda$.) In the vertical direction there is a scaling with factor $\lambda \approx 1.62$, in the horizontal direction the factor is $\lambda^{2} \approx 2.62$.

This process can be extended to find everywhere approximate copies of the vertical strip near $\eta=0$. In Figure 3 a small part of Figure 2 has been enlarged.

2.4. Truncation effect. The presence of gaps in the black band around the horizontal axis in Figure 2 is a truncation effect. We consider it for $0<\eta<1 / 5$. The symmetry $(\eta, \sigma) \mapsto(-\eta,-\sigma)$ and the reasoning that leads to the selfsimilarity explain the other gaps.

For $1<c<N$ and $0<d / c<1 / 5$ we consider $\eta=d / c$ and $\sigma=T(c, d) / c$. We use (3.2), which states that $|T(c, d)| \leq \beta_{5} c$ for some constant $\beta_{5}$, and the reciprocity relation (1.1). Thus

$$
\begin{aligned}
c \sigma & =T(c, d)=-T(d, c)+\frac{3}{\lambda}\left(\frac{c}{d}+\frac{d}{c}+\frac{1}{c d}\right)-5 \geq-\beta_{5} d+\frac{3}{\lambda} \cdot \frac{1}{\eta}-5, \\
\sigma & \geq \frac{3}{c \lambda}\left(\frac{1}{\eta}-\frac{5 \lambda}{3}\right)-\beta_{5} \eta \geq \frac{3}{N \lambda}\left(\frac{1}{\eta}-\frac{5 \lambda}{3}\right)-\beta_{5} \eta .
\end{aligned}
$$

The points $(d / c, T(c, d) / c)$ with $0<d / c<1 / 5$ and $c<N$ stay above the curve $\sigma=\frac{3}{N \lambda}\left(\eta^{-1}-\frac{5 \lambda}{3}\right)-\beta_{5} \eta$. This curve is approximately a piece of a hyperbola; it moves down with increasing $N$.

3. Hecke groups and the associated Dedekind sums. We apply Goldstein's definition of Dedekind sums to the Hecke groups $G_{q}$. This method yields a generalization of the eta function of Dedekind from a study of the Eisenstein series in weight zero.

3.1. Fundamental domain. Hecke [15] gives a fundamental domain for $G_{q}$. It is the region $\{z \in \mathfrak{H}: \operatorname{Re} z \leq \lambda / 2,|z| \geq 1\}$ in the upper 


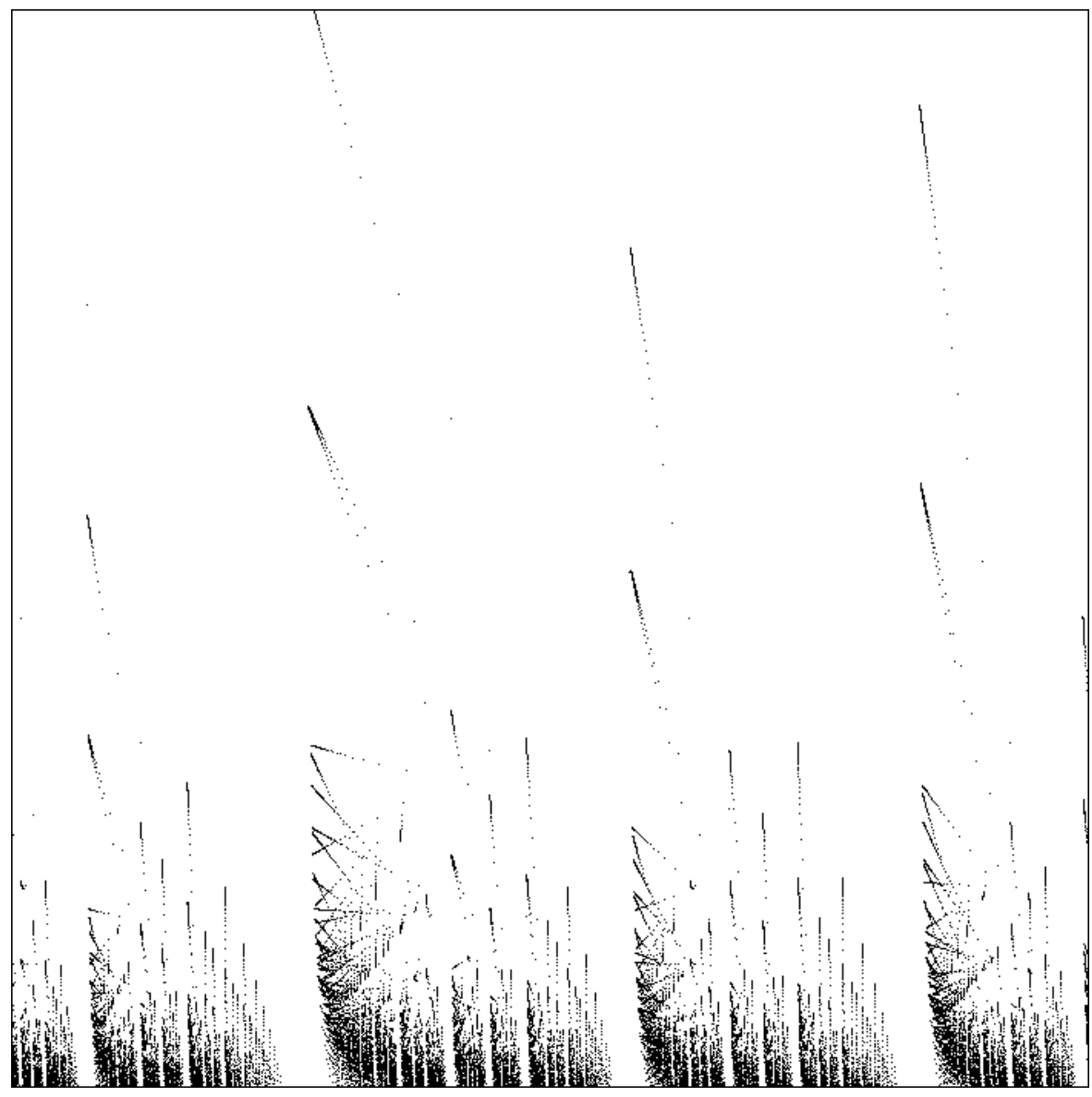

Fig. 3. Enlargement of the region $[0.243,0.485] \times[0.000,0.556]$ of Figure 2

half plane $\mathfrak{H}=\{z \in \mathbb{C}: \operatorname{Im} z>0\}$. It has area $\pi(q-2) / q$. We see that $G_{q}$ has only one cusp.

This fundamental domain is symmetric under reflection in the imaginary axis. This corresponds to the (outer) automorphism $j$ of $G_{q}$ given by the conjugation

$$
j: \gamma \mapsto\left(\begin{array}{cc}
1 & 0 \\
0 & -1
\end{array}\right) \gamma\left(\begin{array}{cc}
1 & 0 \\
0 & -1
\end{array}\right)
$$

which sends both generators to their inverses.

3.2. Automorphic forms. A function $f: \mathfrak{H} \rightarrow \mathbb{C}$ has automorphic transformation behavior of weight $r$ for $G_{q}$ if it satisfies

$$
f\left(\frac{a z+b}{c z+d}\right)=v(\gamma) e^{i r \arg (c z+d)} f(z) \quad \text { for all }\left(\begin{array}{ll}
a & b \\
c & d
\end{array}\right) \in G_{q}
$$


for some map $v: G_{q} \rightarrow \mathbb{C}^{*}$, called the multiplier system of $f$. The argument takes values in $(-\pi, \pi]$. Consider $z \mapsto y^{-r / 2} f(z)$ to get the well known transformation behavior of holomorphic automorphic forms.

To allow non-zero functions $f$ with this behavior, the multiplier system has to satisfy some relations; it is determined by the weight and its values on generators of $G_{q}$.

A function $f$ with automorphic transformation behavior of weight $r$ is a (real analytic) automorphic form if it satisfies

$$
\left(-y^{2} \partial_{y}^{2}-y^{2} \partial_{x}^{2}+i r \partial_{x}\right) f=\beta f
$$

for some $\beta \in \mathbb{C}$, the eigenvalue, and a growth condition at the cusp to be discussed later on. (Here and elsewhere we use $\operatorname{Re} z=x$ and $\operatorname{Im} z=y$ as real coordinates on $\mathfrak{H}$.) This definition is equivalent to Maass' definition in [19].

3.3. Eisenstein series. Examples of automorphic forms are provided by the Eisenstein series

$$
\sum_{\gamma \in G_{q}^{\infty} \backslash G_{q}}\left(\frac{\operatorname{Im}(\gamma \cdot z)}{\lambda}\right)^{s+1 / 2}
$$

where $G_{q}^{\infty}$ is the subgroup of $G_{q}$ generated by $\left(\begin{array}{ll}1 & \lambda \\ 0 & 1\end{array}\right)$ and $\left(\begin{array}{cc}-1 & 0 \\ 0 & -1\end{array}\right)$, and

$$
\left(\begin{array}{ll}
a & b \\
c & d
\end{array}\right) \cdot z=\frac{a z+b}{c z+d}
$$

The discreteness of $G_{q}$ in $\mathrm{SL}_{2}(\mathbb{R})$ implies that any element $\left(\begin{array}{c}\cdots \\ 0\end{array}\right) \in G_{q}$ is an element of $G_{q}^{\infty}$.

The Eisenstein series converges for $\operatorname{Re} s>1 / 2$, and defines, for these values of $s$, an automorphic form of weight 0 , with trivial multiplier system $\gamma \mapsto 1$, and with eigenvalue $1 / 4-s^{2}$; see e.g. [16], Ch. VI, (6.7) on p. 41, Proposition 8.1 on p. 56 and Definition 8.5 on p. 65 . The normalization of the spectral parameter differs from the usual one: $s_{\text {usual }}=s_{\text {here }}+1 / 2$. (The present choice is a bit simpler when Whittaker functions are involved. Moreover, I like to have the unitary principal series to be parametrized by purely imaginary complex numbers.)

3.4. Fourier expansion. Any automorphic form satisfies $f(z+\lambda)=$ $v\left(\begin{array}{ll}1 & \lambda \\ 0 & 1\end{array}\right) f(z)$. Hence there is a Fourier expansion in $x$. For the Eisenstein series this expansion takes the form

$$
e(s ; \lambda z)=y^{s+1 / 2}+c_{0}(s) y^{-s+1 / 2}+\sum_{n \neq 0} c_{n}(s) e^{2 \pi i n x} W_{0, s}(4 \pi|n| y),
$$




$$
\begin{aligned}
& c_{n}(s)=\lambda^{-1-2 s} \varphi_{n}^{0}(s) \cdot \begin{cases}\frac{\pi^{1 / 2} \Gamma(s)}{\Gamma(s+1 / 2)} & \text { if } n=0, \\
\frac{\pi^{s+1 / 2}|n|^{s-1 / 2}}{\Gamma(s+1 / 2)} & \text { if } n \neq 0,\end{cases} \\
& \varphi_{n}^{0}(s)=\sum_{(c, d) \in \mathcal{B}} c^{-1-2 s} e^{2 \pi i n d /(c \lambda)} .
\end{aligned}
$$

$\mathcal{B}$ denotes the set of $(c, d)$ occurring as the lower row of elements of $G_{q}$, with the additional conditions $c>0,-\lambda c / 2<d \leq \lambda c / 2$. The Dirichlet series $\varphi_{n}^{0}(s)$ converge absolutely for $\operatorname{Re} s>1 / 2$. In the modular case, $q=3$, we have $\varphi_{0}^{0}(s)=\zeta(2 s) / \zeta(2 s+1)$ and $\varphi_{n}^{0}(s)=\sigma_{-2 s}(|n|) / \zeta(2 s+1)$ for $n \neq 0$.

In [16], Ch. VI, Theorems 11.6 and 11.8, on p. 128-130, one sees that $e$, and hence the $\varphi_{n}^{0}$ as well, have a meromorphic continuation to $s \in \mathbb{C}$. As $e$ is the unique family of automorphic forms with a Fourier expansion as indicated, it has a functional equation for $s \mapsto-s$. The right-most pole of $e$ is at $s=1 / 2$. It is a first order pole, its residue is the constant function with value $1 /\left(\right.$ area of $\left.G_{q} \backslash \mathfrak{H}\right)=q /(\pi(q-2))$. Further poles in $\operatorname{Re} s \geq 0$ can occur only at points of $(0,1 / 2)$.

3.5. Matrix elements. From the convergence of $\varphi_{0}^{0}(s)$ for $\operatorname{Re} s>1 / 2$ it follows that the positive numbers $c$ that occur in $\left(\begin{array}{l}\cdots \\ c\end{array}\right) \in G_{q}$ form a discrete subset $\mathcal{C}$ of $(0, \infty)$. Left and right multiplication by $\left(\begin{array}{cc}0 & 1 \\ -1 & 0\end{array}\right)$ shows that $\mathcal{C} \cup\{0\} \cup(-\mathcal{C})$ is the set of numbers that occurs at each of the four positions in the matrices in $G_{q}$.

For each $\left(\begin{array}{ll}a & b \\ c & d\end{array}\right) \in G_{q}$ there are \pm 1 and $k \in \mathbb{Z}$ such that $\pm\left(\begin{array}{ll}a & b \\ c & d\end{array}\right)\left(\begin{array}{ll}1 & k \lambda \\ 0 & 1\end{array}\right)$ has its lower row in $\mathcal{B}$. The whole set $\mathcal{B}$, and hence $\mathcal{C}$ as well, can be found recursively:

Let $\mathcal{B}^{+}=\{(c, d) \in \mathcal{B}: d>0\}$ and $\mathcal{B}^{0}=\mathcal{B}^{+} \cup\{(1,0)\}$. As

$$
\left(\begin{array}{cc}
-1 & 0 \\
0 & -1
\end{array}\right)\left(j\left(\begin{array}{ll}
a & b \\
c & d
\end{array}\right)\right)=\left(\begin{array}{cc}
-a & b \\
c & -d
\end{array}\right)
$$

it is clear that we can recover $\mathcal{B}$ from $\mathcal{B}^{+}$. Consider the map $\psi: \mathcal{B}^{+} \rightarrow \mathcal{B}^{0}$ given by $\psi(c, d)=\left(d, d_{1}\right) \in \mathcal{B}^{0}, d_{1} \equiv \pm c \bmod \lambda d \mathbb{Z}$. This corresponds to the following transitions in $G_{q}$ :

$$
\begin{aligned}
& \left(\begin{array}{ll}
a & b \\
c & d
\end{array}\right) \mapsto\left(\begin{array}{ll}
a & b \\
c & d
\end{array}\right)\left(\begin{array}{cc}
0 & -1 \\
1 & 0
\end{array}\right), \\
& \left(\begin{array}{l}
b-a \\
d-c
\end{array}\right) \mapsto\left(\begin{array}{l}
b-a \\
d-c
\end{array}\right)\left(\begin{array}{cc}
1 & r \lambda \\
0 & 1
\end{array}\right) \quad \text { with } r \in \mathbb{Z} \text { suitable, } \\
& \left(\begin{array}{cc}
b & b r \lambda-a \\
d & d r \lambda-c
\end{array}\right) \mapsto-j\left(\begin{array}{cc}
b & b r \lambda-a \\
d & d r \lambda-c
\end{array}\right) \quad \text { if } d r \lambda-c<0 .
\end{aligned}
$$

If $\psi(c, d)=\left(c_{1}, d_{1}\right)$, then $c_{1} \leq \lambda c / 2$. Hence after $\mathcal{O}(\log c)$ iterations of $\psi$ 
we have left the domain $\mathcal{B}^{+}$; remember that $\mathcal{C}$ is discrete. Thus we end up at the element $(1,0)$. By backtracking $\psi$ departing from $(1,0)$, we can construct the whole of $\mathcal{B}^{+}$.

3.6. Generalized eta function. Now I review, for $G_{q}$, Goldstein's introduction of generalized Dedekind sums; see [11]-[13].

The residue of $e$ at $s=1 / 2$ is the constant function with value $q /(\pi(q-2))$. Hence the $\varphi_{n}^{0}$ with $n \neq 0$ are holomorphic at $s=1 / 2$, and

$$
c_{0}(s)=\frac{q}{\pi(q-2)} \cdot \frac{1}{s-1 / 2}+\beta+\mathcal{O}\left(s-\frac{1}{2}\right) \quad \text { for some } \beta \in \mathbb{C} .
$$

Furthermore, $e(s ;-\bar{z})=e(s ; z)$, hence $\varphi_{-n}^{0}(s)=\varphi_{n}^{0}(s)$, with real values for real $s$. Consider the $G_{q}$-invariant function $h$ on $\mathfrak{H}$ given by

$$
h(z)=\lim _{s \rightarrow 1 / 2}\left(e(s ; z)-\frac{q}{\pi(q-2)} \cdot \frac{1}{s-1 / 2}\right) .
$$

It can be written as

$$
h(z)=-\frac{q}{\pi(q-2)} \log \frac{y}{\lambda}+\beta-\frac{4 q}{\pi(q-2)} \operatorname{Re} l(z),
$$

where $l$ is the holomorphic function on $\mathfrak{H}$ given by

$$
l(\lambda z)=l_{q}(\lambda z)=\frac{\pi i z}{4} \cdot \frac{q-2}{q}-\frac{\pi^{2}(q-2)}{2 q \lambda^{2}} \sum_{n=1}^{\infty} \varphi_{n}^{0}\left(\frac{1}{2}\right) e^{2 \pi i n z} .
$$

For $q=3$ we get $l(z)=\log \eta(z)$, the logarithm of the Dedekind eta function.

Let $\gamma=\left(\begin{array}{ll}a & b \\ c & d\end{array}\right) \in \Gamma$. The equality $h(\gamma \cdot z)=h(z)$ may be expressed in terms of $l(z)$ and $\overline{l(z)}$. Bring all holomorphic terms to one side of the equality, and the antiholomorphic terms to the other side. Then both sides have to be equal to the same constant. This yields

$$
l\left(\frac{a z+b}{c z+d}\right)=l(z)+\frac{1}{2} \log (c z+d)+i C(\gamma),
$$

with $C(\gamma)$ a real constant.

One easily sees that $l(z+\lambda)=l(z)+\pi i(q-2) /(4 q)$. For $c \neq 0$ we write the transformation formula as

$$
l\left(\frac{a z+b}{c z+d}\right)=l(z)+\frac{1}{2} \log \left(\frac{c z+d}{i \operatorname{sign} c}\right)+\frac{\pi i}{4 q} \Phi\left(\begin{array}{ll}
a & b \\
c & d
\end{array}\right),
$$

with $\Phi\left(\begin{array}{ll}a & b \\ c & d\end{array}\right) \in \mathbb{R}$. We write $\Phi_{q}$ if the dependence on $q$ has to be emphasized.

3.7. Alternatives. There are Eisenstein series for each even weight. For non-zero weight the meromorphic extension is holomorphic at $s=1 / 2$. Take the value $e_{2}(1 / 2)$ of the Eisenstein series in weight 2. It can be expressed in terms of $l^{\prime}$. One can define $\Phi$ in terms of periods of the differential form associated with $e_{2}(1 / 2)$. In the case of hyperbolic elements in the modular 
group $(q=3)$ the resulting formula is equivalent to (5.28) in [1]. Atiyah derives this description from geometric considerations concerning the bundle of elliptic curves above $G_{3} \backslash \mathfrak{H}$. I do not know whether this approach can be generalized to general Hecke groups $G_{q}$.

If one wants to avoid automorphic forms, one may consider the central extension $0 \rightarrow \mathbb{Z} \rightarrow \widetilde{G}_{q} \rightarrow G_{q} \rightarrow 1$ where $\widetilde{G}_{q}$ is the full original of $G_{q}$ in the universal covering group $\widetilde{\mathrm{SL}_{2}(\mathbb{R})}$ of $\mathrm{SL}_{2}(\mathbb{R})$. It turns out that the group homomorphisms $\widetilde{G}_{q} \rightarrow \mathbb{R}$ form a vector space of dimension 1 . Let $\alpha$ be a basis element. If one takes an obvious section $\sigma: \mathrm{SL}_{2}(\mathbb{R}) \rightarrow \widetilde{\mathrm{SL}_{2}(\mathbb{R})}$ and tries to evaluate $\alpha \circ \sigma$ in terms of the matrix elements of elements of $G_{q}$ one ends up with an expression containing $\Phi$. In another language one can formulate this as " $\Phi$ is needed to describe the connected component of the group of multiplier systems for $G_{q}$ "; see 3.11. To check this consult [23].

3.8. Dedekind sums. Consider the transformation formula for $l(\gamma \cdot z)$ and for $l(\lambda+\gamma \cdot z)$. That gives

$$
\Phi\left(\begin{array}{cc}
a+\lambda c & b+\lambda d \\
c & d
\end{array}\right)=\Phi\left(\begin{array}{ll}
a & b \\
c & d
\end{array}\right)+q-2 .
$$

Hence the quantity

$$
T(c, d)=T_{q}(c, d)=(q-2) \frac{a+d}{\lambda c}-\Phi\left(\begin{array}{ll}
a & b \\
c & d
\end{array}\right)
$$

does not depend on $a$ and $b$. In this definition of the generalized Dedekind "sum" $T(c, d)$ for all lower rows $(c, d)$ occurring in $G_{q}$ there is the freedom of a multiplicative factor. The present choice seems a sensible one; $\lambda$ times it might be as good.

From the transformation formula one obtains more properties of $\Phi$ and $T$ (see the table below); we take $\gamma=\left(\begin{array}{ll}a & b \\ c & d\end{array}\right) \in G_{q}$ with $c \neq 0$. These relations imply that $\Phi$ has integral values.

\begin{tabular}{|l|c|c|}
\hline 1 & $\Phi\left(\begin{array}{ll}-a & -b \\
-c & -d\end{array}\right)=\Phi\left(\begin{array}{ll}a & b \\
c & d\end{array}\right)$ & $T(-c,-d)=T(c, d)$ \\
\hline 2 & $\Phi\left(\begin{array}{ll}0 & -1 \\
1 & 0\end{array}\right)=0$ & $T(1,0)=0$ \\
\hline 3 & $\Phi\left(\begin{array}{ll}a & a \lambda+b \\
c & c \lambda+d\end{array}\right)=\Phi\left(\begin{array}{ll}a & b \\
c & d\end{array}\right)+q-2$ & $T(c, c \lambda+d)=T(c, d)$ \\
\hline 4 & $\Phi\left(\begin{array}{cc}-a & b \\
c & -d\end{array}\right)=-\Phi\left(\begin{array}{ll}a & b \\
c & d\end{array}\right)$ & $-T(c,-d)=T(c, d)$ \\
\hline 5 & $\Phi\left(\begin{array}{ll}d & b \\
c & a\end{array}\right)=\Phi\left(\begin{array}{ll}a & b \\
c & d\end{array}\right)$ & $T(c, a)=T(c, d)$ \\
\hline & Reciprocity law. For $c, d>0$ \\
& $\Phi\left(\begin{array}{cc}-b & -a \\
d & c\end{array}\right)=-\Phi\left(\begin{array}{ll}a & b \\
c & d\end{array}\right)+q$ & $T(c, d)+T(d, c)$ \\
& & $(q-2) \frac{c^{2}+d^{2}+1}{\lambda c d}-q$ \\
\hline
\end{tabular}


To prove (2), take $z=i$ in the transformation formula. For (4) use $\overline{l(z)}=l(-\bar{z})$. For (5) apply the transformation formula to $\gamma^{-1} \cdot \gamma \cdot z$; after that use (1) and (4).

Formula (6) is the reciprocity law. Take $z=-1 / z=i$, and apply the transformation formula in two ways to get

$$
\frac{1}{2} \log (c-d i)+\frac{\pi i}{4 q} \Phi\left(\begin{array}{ll}
a & b \\
c & d
\end{array}\right)=\frac{1}{2} \log (c i+d)+\frac{\pi i}{4 q} \Phi\left(\begin{array}{ll}
b & -a \\
d & -c
\end{array}\right) .
$$

This implies

$$
\Phi\left(\begin{array}{ll}
b & -a \\
d & -c
\end{array}\right)=\Phi\left(\begin{array}{ll}
a & b \\
c & d
\end{array}\right)-q
$$

Now use (4).

3.9. Three Dedekind sums. For $\gamma_{j}=\left(\begin{array}{ll}a_{j} & b_{j} \\ c_{j} & d_{j}\end{array}\right) \in G_{q}, c_{j}>0$, for $j=1,2,3$, with $\gamma_{1} \gamma_{2}= \pm \gamma_{3}$, we apply the transformation formula to $l\left(\gamma_{3} \cdot z\right)=l\left(\gamma_{1} \gamma_{2} \cdot z\right)$ to find

$$
\begin{aligned}
\Phi\left(\gamma_{3}\right) & =\Phi\left(\gamma_{1}\right)+\Phi\left(\gamma_{2}\right) \mp q, \\
T\left(c_{3}, d_{3}\right) & =T\left(c_{1}, d_{1}\right)+T\left(c_{2}, d_{2}\right) \mp \frac{q-2}{\lambda}\left(\frac{c_{1}}{c_{2} c_{3}}+\frac{c_{2}}{c_{1} c_{3}}+\frac{c_{3}}{c_{1} c_{2}}\right) \pm q .
\end{aligned}
$$

This result is needed to complete the proof of Proposition 1.7.

\subsection{Estimate.}

$$
T(c, d)=\mathcal{O}(c) .
$$

It suffices to show that $|T(c, d)| \leq \beta c$ for all $(c, d) \in \mathcal{B}^{0}$, for some $\beta>0$. We shall get $\beta=3 q /(2-\lambda)$.

Of course we apply induction on the size of $c$. As $T(1,0)=0$, the start is no problem. Consider $(c, d) \in \mathcal{B}^{+}$. If we can show that the right hand side in the reciprocity law satisfies $\mid$ RHS $\mid \leq \alpha c$ for some positive $\alpha$, then we get by induction $|T(c, d)| \leq \beta d+\alpha c \leq(\lambda \beta / 2+\alpha) c$, and $|T(c, d)| \leq \beta c$ if we choose $\beta=2 \alpha /(2-\lambda)$.

We use the fact that 1 is the minimal element of $\mathcal{C}$, and hence $1 \leq d \leq$ $\lambda c / 2$. We get

$$
\mathrm{RHS}=\frac{q-2}{\lambda}\left(\frac{c}{d}+\frac{d}{c}+\frac{1}{c d}\right)-q \leq \frac{q-2}{\lambda}\left(c+\frac{\lambda}{2}+1-\lambda\right) \leq \frac{3}{2} q c,
$$

and

$$
\text { RHS } \geq-q \geq-\frac{3}{2} q c .
$$

This leads to $\alpha=3 q / 2$ and $\beta=3 q /(2-\lambda)$. 
3.11. Multiplier systems. Let $r \in \mathbb{C}$. The function $\eta_{r}(z)=e^{2 r l(z)}$ is a holomorphic function on $\mathfrak{H}$, and satisfies, for each $\gamma \in G_{q}$,

$$
\eta_{r}(\gamma \cdot z)=v_{r}(\gamma)(c z+d)^{r} \eta_{r}(z)
$$

where $v_{r}$ is the multiplier system that satisfies

$$
\begin{aligned}
v_{r}\left(\begin{array}{ll}
a & b \\
c & d
\end{array}\right) & =e^{-\pi i r / 2+\pi i r(q-2)(a+d) /(2 q \lambda c)-\pi i r T(c, d) /(2 q)} \\
& =\exp \left(\pi i r\left(\frac{1}{2 q} \Phi\left(\begin{array}{ll}
a & b \\
c & d
\end{array}\right)-\frac{1}{2}\right)\right)
\end{aligned}
$$

if $c>0$. The logarithm of $c z+d$ is taken with respect to the argument convention $-\pi<\arg (c z+d) \leq \pi$. We see that $z \mapsto y^{r / 2} \eta_{r}(z)$ has automorphic transformation behavior of weight $r$. The function $z \mapsto y^{r / 2} \eta_{r}(z)$ is an automorphic form of weight $r$, with eigenvalue $\frac{r}{2}\left(1-\frac{r}{2}\right)$ for the multiplier system $v_{r}$. All other multiplier systems for weight $r$ are of the form $\chi \cdot v_{r}$, with $\chi$ a character of $G_{q} /\{ \pm I\}$. In the modular case all possible characters of $G_{3} /\{ \pm I\}$ are of the form $v_{n}$ with $n \in 2 \mathbb{Z}$. For general $G_{q}$, the description of all characters is slightly more complicated.

3.12. Congruence cases. We have already remarked that $G_{3}$ is the modular group $\mathrm{SL}_{2}(\mathbb{Z})$, that $l_{3}$ is the logarithm of the eta function of Dedekind, and that $T_{3}(c, d)=12 S(d, c)$. We now consider the cases $q=2 k, k=2,3$, with $\lambda=\sqrt{k}$.

For these values of $k$ the subgroups

$$
G_{0}(k)=\left\{\left(\begin{array}{ll}
a & b \\
c & d
\end{array}\right) \in \mathrm{SL}_{2}(\mathbb{Z}): c \equiv 0 \bmod k\right\}
$$

are generated by $\left(\begin{array}{ll}1 & 1 \\ 0 & 1\end{array}\right)$ and $\left(\begin{array}{ll}1 & 0 \\ k & 1\end{array}\right)$. As $\left(\begin{array}{ll}1 & 0 \\ \lambda & 1\end{array}\right) \in G_{q}$ for all $q \geq 3$, we get for $q=2 k$ the conjugate group

$$
\Delta_{2 k}=\left(\begin{array}{cc}
\sqrt{k} & 0 \\
0 & 1
\end{array}\right) G_{0}(k)\left(\begin{array}{cc}
1 / \sqrt{k} & 0 \\
0 & 1
\end{array}\right)
$$

inside $G_{2 k}$. From a computation of the areas of fundamental domains it follows that $\Delta_{2 k}$ has index 2 in $G_{2 k}$. As $\left(\begin{array}{cc}0 & 1 \\ -1 & 0\end{array}\right) \notin \Delta_{2 k}$, we have $G_{2 k}=$ $\Delta_{2 k} \cup\left(\begin{array}{cc}0 & 1 \\ -1 & 0\end{array}\right) \Delta_{2 k}$.

The fact that $G_{2 k}$ is conjugate to well known congruence subgroups of the modular group leads to an explicit description of $l_{2 k}$. It suffices to exhibit a holomorphic function $m$ on $\mathfrak{H}$ with the same transformation behavior under the generators of $G_{2 k}$ as that of $l_{2 k}$, and such that $m(z)=l_{2 k}(z)+o(1)$ $(y \rightarrow \infty)$. It turns out that $m(z)=\frac{1}{2} \log \eta(z \sqrt{k})+\frac{1}{2} \log \eta(z / \sqrt{k})$ satisfies 
these conditions. This implies for $\left(\begin{array}{ll}a & b \\ c & d\end{array}\right) \in \Delta_{2 k}, c \neq 0$,

$$
\begin{aligned}
\Phi_{2 k}\left(\begin{array}{ll}
a & b \\
c & d
\end{array}\right) & =\frac{k}{3}\left(\Phi_{3}\left(\begin{array}{cc}
a & b / \sqrt{k} \\
c \sqrt{k} & d
\end{array}\right)+\Phi_{3}\left(\begin{array}{cc}
a & b \sqrt{k} \\
c / \sqrt{k} & d
\end{array}\right)\right), \\
T_{2 k}(c, d) & =4 k(S(d, c \sqrt{k})+S(d, c / \sqrt{k})),
\end{aligned}
$$

and for $\left(\begin{array}{ll}a & b \\ c & d\end{array}\right) \in\left(\begin{array}{cc}0 & 1 \\ -1 & 0\end{array}\right) \Delta_{2 k}$,

$$
\begin{aligned}
\Phi_{2 k}\left(\begin{array}{ll}
a & b \\
c & d
\end{array}\right) & =\frac{k}{3}\left(\Phi_{3}\left(\begin{array}{cc}
a / \sqrt{k} & b \\
c & d \sqrt{k}
\end{array}\right)+\Phi_{3}\left(\begin{array}{cc}
a \sqrt{k} & b \\
c & d / \sqrt{k}
\end{array}\right)\right), \\
T_{2 k}(c, d) & =4 k(S(d \sqrt{k}, c)+S(d / \sqrt{k}, c)) .
\end{aligned}
$$

4. The structure of $\beta\left(G_{q}^{0}\right)$. In this section we prove Proposition 1.9. To do that we prove Lemma 4.1 below. Proposition 1.7 implies that the other inclusion in Proposition 1.9 can be arranged by adapting $X$.

Remember that $\mathcal{P} \subset \mathbb{R}^{3}$ is the plane $\{(\xi, \eta, \sigma): \sigma=0\}$, and that $\mathcal{L}$ is the set of limit points $\left(a_{1} / c_{1}, d_{2} / c_{2}, \pm(q-2) / c_{1} c_{2}\right)$, with $\left(\begin{array}{ll}a_{j} & b_{j} \\ c_{j} & d_{j}\end{array}\right) \in G_{q}, c_{j}>0$. We use $A^{ \pm}(p, X)$ and $U_{\varepsilon}(p)$ as defined in 1.8.

4.1. Lemma. For each $p=(\xi, \eta, \sigma) \in \mathbb{R}^{3}$ with $\mp \sigma>0$, there are $\varepsilon>0$ and $X>1$ such that the set

$$
\left\{\beta\left(\begin{array}{ll}
a & b \\
c & d
\end{array}\right) \in U_{\varepsilon}(p):\left(\begin{array}{ll}
a & b \\
c & d
\end{array}\right) \in G_{q}^{0}, c>X\right\}
$$

is contained in $A^{ \pm}(p, X)$ if $p \in \mathcal{L}$, and is empty otherwise.

4.2. Plan of the proof. For the cases $\eta=0$ and $|\sigma|$ large the statement in the lemma is proved directly; see 4.3 and Lemma 4.4. To get the other cases we show in 4.5 and 4.7 that if the statement in the lemma holds for $p$, it holds for some other $p_{1}$ as well. In 4.8 we shall see that this suffices to reach all $p$.

4.3. Large $\sigma$. According to $(3.2)$ all $T(d, c) / c$ are contained in some finite interval $\left[-\beta_{q}, \beta_{q}\right]$. Hence the statement in Lemma 4.1 holds if $|\sigma|>\beta_{q}$.

4.4. Lemma. The statement in Lemma 4.1 holds for $p=(\xi, 0, \sigma)$ with $\sigma \neq 0$.

Proof. The set $\{|\sigma-(q-2) /(\lambda d)|:|d| \in \mathcal{C}\}$ has a minimal element $\alpha$. For $\gamma=\left(\begin{array}{ll}a & b \\ c & d\end{array}\right) \in G_{q}^{0}$ with $\beta(\gamma) \in U_{\varepsilon}(p), c>X$, and with $\varepsilon>0$ and $X>1$ to be fixed later, we obtain from the reciprocity law

$$
\begin{aligned}
\left|\frac{T(c, d)}{c}-\frac{q-2}{\lambda d}\right| & =\left|-\frac{d}{c} \cdot \frac{T(|d|, c)}{|d|}+\frac{q-2}{\lambda}\left(\frac{d}{c^{2}}+\frac{1}{c^{2} d}\right)-\frac{q \operatorname{sign} d}{c}\right| \\
& \leq \varepsilon \beta_{q}+\frac{(q-2) \varepsilon}{\lambda X}+\frac{q-2}{\lambda X^{2}}+\frac{q}{X}=: C(\varepsilon, X) .
\end{aligned}
$$


We conclude

$$
\alpha \leq\left|\sigma-\frac{q-2}{\lambda d}\right|<C(\varepsilon, X)+\varepsilon .
$$

If $\alpha>0$, we can choose $\varepsilon$ and $X$ such that $\gamma$ as above cannot be found. Then the set in Lemma 4.1 is empty, and we are done.

If $\alpha=0$ we know that $\sigma=\mp(q-2) /\left(\lambda c_{1}\right)$ for some $c_{1} \in \mathcal{C}$. A suitable choice of $\varepsilon$ and $X$ ensures that $d=\mp c_{1}$ for all $\gamma=\left(\begin{array}{ll}a & b \\ c & d\end{array}\right) \in U_{\varepsilon}(p)$ with $c>X$. For such $\gamma$ we have

$$
\left|\xi \pm \frac{b}{c_{1}}\right| \leq \varepsilon+\left|\frac{a}{c} \pm \frac{b}{c_{1}}\right|=\varepsilon+\frac{1}{\left|c c_{1}\right|} \leq \varepsilon+c_{1}^{-1} X^{-1} .
$$

As the set of $\mp b / c_{1}$ with $c_{1}$ fixed and $\left(\begin{array}{c}b \\ \cdot \mp c_{1}\end{array}\right) \in G_{q}$ is discrete, a suitable choice of $\varepsilon$ and $X$ assures us that the existence of $\gamma$ as above implies that it has the form $\gamma=\left(\begin{array}{l}a \mp a_{1} \\ c \mp c_{1}\end{array}\right)$, with $a_{1}$ fixed, and that $\xi=a_{1} / c_{1}$. Choose $\gamma_{1}=$

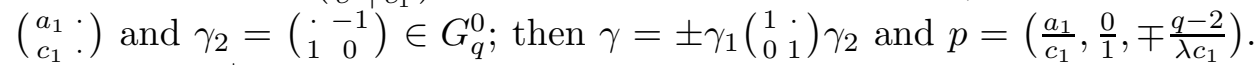
Hence $\gamma \in A^{ \pm}(p, X)$.

4.5. Translation. Consider the maps $T_{n}:(\xi, \eta, \sigma) \mapsto(\xi, \eta+n \lambda, \sigma)$ in $\mathbb{R}^{3}$. As

$$
\beta\left(\gamma\left(\begin{array}{cc}
1 & n \lambda \\
0 & 1
\end{array}\right)\right)=T_{n} \beta(\gamma) \quad \text { and } \quad A^{ \pm}\left(T_{n} p, X\right)=T_{n} A^{ \pm}(p, N)
$$

the statement of Lemma 4.1 is true for all $T_{n} p$, if it holds for one of them. We can use the same $\varepsilon$ and $X$.

4.6. Involution. Let $W(\xi, \eta, \sigma)=(\xi,-1 / \eta, \sigma /|\eta|)$. This is an involution in $\left\{(\xi, \eta, \sigma) \in \mathbb{R}^{3}: \eta \neq 0\right\}$.

Remark that $T_{1}$ and $W$ generate a partial action of $G_{q} /\{ \pm I\}$ in $\mathbb{R}^{3}$. The image of $\beta$ is approximately invariant under this action. To see this we show that $\beta\left(\gamma\left(\begin{array}{cc}0 & -1 \\ 1 & 0\end{array}\right)\right) \approx W \beta(\gamma)$. Let $\gamma=\left(\begin{array}{ll}a & b \\ c & d\end{array}\right) \in G_{q}^{0}$ with $c$ large; put $\widetilde{\gamma}=\gamma\left(\begin{array}{cc}0 & -1 \\ 1 & 0\end{array}\right)$, then $\widetilde{\gamma} \in G_{q}^{0}$ as well.

$$
\begin{aligned}
\beta(\widetilde{\gamma}) & =\beta\left(\begin{array}{c}
b \operatorname{sign} d-a \operatorname{sign} d \\
|d|
\end{array}\right) \\
& =\left(\frac{b}{d},-\frac{c}{d}, \frac{-T(|d|, c)}{d}\right) \\
& =\left(\frac{a}{c}-\frac{1}{c d}, \frac{-1}{d / c}, \frac{T(c, d)}{|d|}-\frac{q-2}{\lambda} \cdot \frac{c}{d|d|}+\mathcal{O}\left(\frac{1}{c}+\frac{1}{|d|}\right)\right) \\
& =W \beta(\gamma)-\frac{q-2}{\lambda}\left(0,0, \frac{c}{d|d|}\right)+\mathcal{O}\left(\frac{1}{c}+\frac{1}{|d|}\right) .
\end{aligned}
$$

If $d / c$ is near $\eta \neq 0$, then $1 / d=\mathcal{O}_{\eta}(1 / c)$, and

$$
\beta(\widetilde{\gamma})=W \beta(\gamma)+\mathcal{O}_{\eta}(1 / c) .
$$


4.7. Lemma. If the statement in Lemma 4.1 holds for $p=(\xi, \eta, \sigma)$, with $\eta \neq 0, \mp \sigma>0$, then it holds for $W p$.

P r o of. Consider $\gamma=\left(\begin{array}{ll}a & b \\ c & d\end{array}\right) \in G_{q}^{0}$ with $c>X$ and $\beta(\gamma) \in U_{\varepsilon}(W p)$, with $\varepsilon>0$ and $X>1$ to be specified later on. Equation (4.1) and the continuity of $W$ at $W p$ allows us to choose $\varepsilon$ and $X$ in such a way that we can apply Lemma 4.1 to $\widetilde{\gamma}=\operatorname{sign}(d) \gamma\left(\begin{array}{cc}0 & -1 \\ 1 & 0\end{array}\right)$. This means that $\beta(\widetilde{\gamma}) \in A^{ \pm}(p, \widetilde{X})$ if $p \in \mathcal{L}$, and that such $\widetilde{\gamma}$ are impossible if $p \notin \mathcal{L}$. As $p \in \mathcal{L}$ and $W p \in \mathcal{L}$ are easily seen to be equivalent statements, this finishes the case $p \notin \mathcal{L}$.

Let

$$
p=\left(\frac{a_{1}}{c_{1}}, \frac{d_{2}}{c_{2}}, \mp \frac{q-2}{\lambda c_{1} c_{2}}\right) \quad \text { with } \gamma_{j}=\left(\begin{array}{c}
a_{j} b_{j} \\
c_{j} d_{j}
\end{array}\right) \in G_{q}^{0}, \quad c_{j}>0 .
$$

Put $\zeta=\operatorname{sign} d_{2}$. Then

$$
W p=\left(\frac{a_{1}}{c_{1}}, \frac{\widetilde{d}_{2}}{\widetilde{c}_{2}}, \mp \frac{q-2}{\lambda c_{1} \widetilde{c}_{2}}\right) \quad \text { with } \widetilde{\gamma}_{2}=\left(\begin{array}{cc}
\widetilde{a}_{2} & \widetilde{b}_{2} \\
\widetilde{c}_{2} & \widetilde{d}_{2}
\end{array}\right)=\left(\begin{array}{cc}
\zeta b_{2} & -\zeta a_{2} \\
\left|d_{2}\right| & -\zeta c_{2}
\end{array}\right) .
$$

We have arranged $\varepsilon$ and $X$ so that $\beta(\widetilde{\gamma}) \in A^{ \pm}(W p, \widetilde{X})$, for $\widetilde{X}$ suitable. Hence $\widetilde{\gamma}= \pm \gamma_{1}\left(\begin{array}{cc}1 & r \lambda \\ 0 & 1\end{array}\right) \gamma_{2}$ for some $r \in \mathbb{Z}$. This gives $\gamma= \pm \operatorname{sign}(d) \zeta \gamma_{1}\left(\begin{array}{cc}1 & r \lambda \\ 0 & 1\end{array}\right) \widetilde{\gamma}_{2}$. As we started with $c>X$ and $\left|\frac{d}{c}-\frac{d_{1}}{c_{1}}\right|<\varepsilon$, we may arrange $\varepsilon$ such that sign $d=\zeta$. Hence $\beta(\gamma) \in A^{ \pm}(W p, X)$.

4.8. Induction. We start with $p=(\xi, \eta, \sigma), \sigma \neq 0$. If $\eta=0$ or $|\sigma|>\beta_{q}$, the proof of the statement in Lemma 4.1 is given in Lemma 4.4 or in 4.3. Otherwise we reduce the proof to that for another $p_{1}$ by 4.5 and Lemma 4.7: take $n \in \mathbb{Z}$ such that $\eta_{1}=\eta+n l \in(-\lambda / 2, \lambda / 2]$, and put $p_{1}=W T_{n} p=$ $\left(\xi,-1 / \eta_{1}, \sigma /\left|\eta_{1}\right|\right)$. As $\left|\sigma / \eta_{1}\right| \geq 2|\sigma| / \lambda$, iteration of this process will bring us into the reach of 4.3, unless $\eta$ becomes 0 at a certain stage; then Lemma 4.4 can be applied.

5. Poincaré series. To prove the distribution results we shall use the fact that Dedekind sums describe multiplier systems of automorphic forms.

In this section we consider Poincaré series in their domain of convergence, and describe their Fourier coefficients.

5.1. Automorphic forms. We restrict our attention to automorphic forms of weight $r$, eigenvalue $1 / 4-s^{2}$ and multiplier system $v_{r}$ (see 3.11).

For $\operatorname{Re} s \geq 0$ and $|\operatorname{Re} r|<4 q /(q-2)$ we impose a growth condition at the cusp by prescribing a Fourier series of a special form. Let $f$ satisfy the conditions in 3.2 , with $\beta=1 / 4-s^{2}$. The transformation behavior $f(z+\lambda)=$ $e^{\pi i r(q-2) /(2 q)} f(z)$ implies that $f$ has a Fourier series expansion $f(\lambda z)=$ $\sum_{n} f_{n}(z)$, where $n \equiv(q-2) r /(4 q) \bmod 1$, and $f_{n}(x+i y)=e^{2 \pi i n x} f_{n}(i y)$. The functions $y \mapsto f_{n}(i y)$ satisfy second order linear differential equations that depend on $n$ and $s$. It is not possible to give a basis of the solution space 
that is useful for all $(r, s)$. But for $\operatorname{Re} n \neq 0$ a one-dimensional subspace of solutions stands out by an exponentially decreasing behavior at infinity; it is spanned by

$$
\omega^{n}(r, s ; z)=e^{2 \pi i n x} W_{ \pm r / 2, s}( \pm 4 \pi n y) \quad \text { for } \pm \operatorname{Re} n>0 .
$$

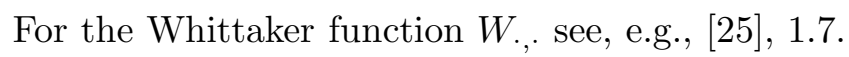

The growth condition we impose on automorphic forms is: $f_{n}$ is a multiple of $\omega^{n}(r, s)$ for all but a finite number of $n$. This growth condition allows exponential growth at the cusp. Usually one allows only polynomial growth.

5.2. The basis element $\omega^{n}(r, s ; z)$ is holomorphic in $(r, s)$; here and in the sequel we tacitly assume that $n$ varies with $r$ such that $n-(q-2) r /(4 q)$ stays constant (with an integral value). The following basis element $\mu^{n}(r, s)$ is meromorphic in $(r, s)$, with its singularities at the lines $s=-l / 2$, $l \in \mathbb{N}:$

$$
\mu^{n}(r, s ; z)=y^{s+1 / 2} e^{2 \pi i n z}{ }_{1} F_{1}\left[\begin{array}{c}
1 / 2+s-r / 2 \\
1+2 s
\end{array} \mid 2 \pi n y\right] .
$$

It is characterized by its behavior $\mu^{n}(r, s ; i y) \sim y^{s+1 / 2}$ for $y \downarrow 0$. For general values of $(r, s)$ it increases exponentially as $y \rightarrow \infty$. Note that $\mu^{0}(0, s ; z)=$ $y^{s+1 / 2}$, occurring in the Fourier expansion of the Eisenstein series in 3.4.

For general values of $(r, s)$ the functions $\omega^{n}(r, s)$ and $\mu^{n}(r, s)$ span the space of possible Fourier terms of order $n$.

5.3. Poincaré series. For $r \in \mathbb{R}, m \equiv(q-2) r /(4 q) \bmod 1$, and $\operatorname{Re} s>1 / 2$, the absolutely converging Poincaré series

$p^{m}(r, s ; z)=\sum_{\gamma \in G_{q}^{\infty} \backslash G_{q}} v_{r}(\gamma)^{-1} e^{-i r \arg (c z+d)} \mu^{m}(r, s ;(\gamma \cdot z) / \lambda) \quad$ with $\gamma=\left(\begin{array}{ll}a & b \\ c & d\end{array}\right)$

defines an automorphic form $p^{m}(r, s)$ of weight $r$, with eigenvalue $1 / 4-s^{2}$ and multiplier system $v_{r}$. For $r=0$ these are the Poincaré series studied by Neunhöffer [22]. Even more general Poincaré series than the present ones are discussed in [16], Ch. IX, $\S 5$, see p. 355. The Eisenstein series discussed in 3.3 is given by $e(s)=p^{0}(0, s)$.

Define the Fourier coefficients $c_{n}^{m}(r, s)$ by

$$
p^{m}(r, s ; \lambda z)=\mu^{m}(r, s ; z)+\sum_{n} c_{n}^{m}(r, s) \eta^{n}(r, s ; z),
$$

with $\eta^{n}(r, s)=\omega^{n}(r, s)$ if $n \neq 0$, and $\eta^{0}(r, s)=\mu^{0}(r,-s)$. The principle of computing $c_{n}^{m}(r, s)$ is well known. In the same way as in Proposition 2.16 in [5] one arrives at the following description. Write 


$$
m=\mu+\frac{r(q-2)}{4 q} \quad \text { and } \quad n=\nu+\frac{r(q-2)}{4 q} .
$$

(One may also use Proposition 5.2.9 in [9]. Then one should note that the roles of $n$ and $m$ have been reversed, and that the factor $\lambda^{-1-2 s}$ has been moved.)

$$
\begin{aligned}
& c_{n}^{m}(r, s)=G_{r}(n, m, s) \varphi_{\nu}^{\mu}(r, s), \\
& G_{r}(n, m, s)=e^{-\pi i r / 2} \lambda^{-1-2 s} \\
& \times \begin{cases}\frac{\pi 2^{1-2 s} \Gamma(2 s)}{\Gamma(1 / 2+s+r / 2) \Gamma(1 / 2+s-r / 2)} & \text { if } n=0, \\
\frac{\pi^{s+1 / 2}|n|^{s-1 / 2}}{\Gamma(1 / 2+s \pm r / 2)} & \text { if } \pm n>0,\end{cases} \\
& \varphi_{\nu}^{\mu}(r, s)=\sum_{(c, d) \in \mathcal{B}} J(n, m, s ; c) v_{r}\left(\begin{array}{ll}
a & b \\
c & d
\end{array}\right)^{-1} e^{2 \pi i(m a+n d) /(\lambda c)}, \\
& J(n, m, s ; c)= \begin{cases}c^{-1-2 s} & \text { if } m n=0, \\
c^{-1} J_{2 s}\left(\frac{4 \pi \sqrt{m n}}{\lambda c}\right) \frac{\Gamma(1+2 s)}{(2 \pi / \lambda)^{2 s}(m n)^{s}} & \text { if } m n>0, \\
c^{-1} I_{2 s}\left(\frac{4 \pi \sqrt{|m n|}}{\lambda c}\right) \frac{\Gamma(1+2 s)}{(2 \pi / \lambda)^{2 s}|m n|^{s}} & \text { if } m n<0 .\end{cases}
\end{aligned}
$$

In the sum over $\mathcal{B}$ the choice of $a, b$ does not influence the result.

The $\varphi_{n}^{0}(s)$ in 3.4 are the present $\varphi_{\nu}^{0}(0, s)$.

5.4. Symmetry. $\varphi_{\nu}^{\mu}(0, s)=\varphi_{\mu}^{\nu}(0, s)$. To see this, note that for $(c, d) \in \mathcal{B}$ there is a unique $\gamma=\left(\begin{array}{ll}a & b \\ c & d\end{array}\right) \in G_{q}$ with $-l c / 2<a \leq \lambda c / 2$. The map $\gamma \mapsto j\left(\gamma^{-1}\right)$ induces an involution in $\mathcal{B}$ that gives the symmetry.

5.5. Dirichlet series with Dedekind sums. In the remainder of this section we relate the $\varphi_{\nu}^{\mu}$ to the following series:

$$
\Delta_{k}(\nu, \mu, w)=\sum_{(c, d) \in \mathcal{B}} c^{-w}\left(\frac{T(c, d)}{c}\right)^{k} e^{2 \pi i(\mu a+\nu d) /(\lambda c)},
$$

with $\nu, \mu, k \in \mathbb{Z}, k \geq 0$. In view of the estimate in (3.2) this converges absolutely and defines a holomorphic function for $\operatorname{Re} w>2$. The choice

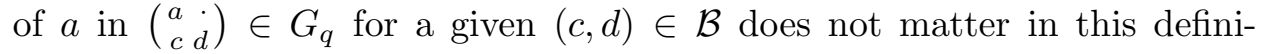
tion.

5.6. Proposition. Let $\nu, \mu \in \mathbb{Z}, l \in \mathbb{N}, l \geq 2$. For fixed $s \in \mathbb{C}$ with $\sigma=\operatorname{Re} s \in(l / 2,(l+1) / 2)$, we have 


$$
\begin{aligned}
e^{-\pi i r / 2} \varphi_{\nu}^{\mu}(r, s) & \\
\quad= & \sum_{h=0}^{l-1} r^{h} \sum_{p=0}^{\infty} \sum_{k=0}^{h} \beta_{p, k}^{h}(\nu, \mu, s) \Delta_{k}(\nu, \mu, 1+2 s+2 p-k)+\mathcal{O}\left(r^{2 \sigma-1}\right)
\end{aligned}
$$

as $r \downarrow 0$, with

$$
\begin{aligned}
& \beta_{p, k}^{h}(\nu, \mu, s) \\
& \quad=\frac{(2 \pi i)^{k+2 p}(q-2)^{h-k}}{(4 q)^{h} \lambda^{2 p} k ! p !(1+2 s)_{p}} \sum_{j_{1}, j_{2} \in[0, p], j_{1}+j_{2}=h-k}\left(\begin{array}{c}
p \\
j_{1}
\end{array}\right)\left(\begin{array}{c}
p \\
j_{2}
\end{array}\right) \mu^{p-j_{1}} \nu^{p-j_{2}} .
\end{aligned}
$$

Remark. This result extends Lemma 3.7 in [5]. There $q=3$ and $\mu=0$; as $\beta_{p, k}^{h}(\nu, 0, s)=0$ for $k+p>h$, the sum expressing $\varphi_{\nu}^{0}$ in the $\Delta_{k}$ is finite if $\mu=0$. In this sense the dependence on $a / c$ makes the proof of the distribution results more difficult than that in loc. cit.

The proof is given in the remainder of this section.

5.7. Splitting up the Bessel functions. In the sequel $m$ and $n$ vary with $r$, whereas $\mu$ and $\nu$ stay fixed. The sign $\eta$ of

$$
m n=\left(\mu+\frac{r(q-2)}{4 q}\right)\left(\nu+\frac{r(q-2)}{4 q}\right)
$$

does not change for small positive $r$. Put $J_{2 s}^{1}=J_{2 s}$ and $J_{2 s}^{-1}=I_{2 s}$.

$$
\begin{aligned}
J(n, m, s ; c) & =c^{-1} J_{2 s}^{\eta}\left(\frac{4 \pi \sqrt{\eta m n}}{\lambda c}\right) \frac{\Gamma(1+2 s)}{(2 \pi / \lambda)^{2 s}(\eta m n)^{s}} \\
& =\sum_{p=0}^{\infty} c^{-1-2 s-2 p} \frac{\left(-4 \pi^{2} m n\right)^{p}}{p !(1+2 s)_{p} \lambda^{2 p}} .
\end{aligned}
$$

The multiplier factor and the exponential in the series for $\varphi_{\nu}^{\mu}(r, s)$ have absolute value one. We can interchange the order of summation with respect to $(c, d)$ and $p$. This gives

$$
\begin{aligned}
\varphi_{\nu}^{\mu}(r, s) & =e^{\pi i r / 2} \sum_{p=0}^{\infty} \alpha_{p}(n, m, s) \Phi_{\nu}^{\mu}(r, s+p), \\
\alpha_{p}(n, m, s) & =\frac{\left(-4 \pi^{2} m n\right)^{p}}{p !(1+2 s)_{p} \lambda^{2 p}}, \\
\Phi_{\nu}^{\mu}(r, u) & =\sum_{(c, d) \in \mathcal{B}} c^{-2 u-1} e^{\pi i r T(c, d) /(2 q)} e^{2 \pi i(\mu a+\nu d) /(\lambda c)} .
\end{aligned}
$$

The dependence of $\alpha_{p}$ on $r$ is hidden in $m$ and $n$. 
5.8. Lemma. For $\operatorname{Re} u \geq v>1 / 2$,

$$
\sum_{(c, d) \in \mathcal{B}, c \geq X} c^{-1-2 u}=\mathcal{O}_{v}\left(X^{1-2 \operatorname{Re} u}\right) \quad(X \rightarrow \infty) .
$$

For $-2<\operatorname{Re} w<-1$,

$$
\sum_{(c, d) \in \mathcal{B}, c<X} c^{-w}=\mathcal{O}\left(X^{2-\operatorname{Re} w}\right) \quad(X \rightarrow \infty) .
$$

Rem ark. For $q=3$ this is easy.

Proof of Lemma 5.8. We use the fact that $\varphi_{0}^{0}(0, s)$ has a meromorphic extension to $s \in \mathbb{C}$, with as right-most singularity a first order pole at $s=1 / 2$ with residue $q \lambda^{2} /\left(\pi^{2}(q-2)\right)$. For $\operatorname{Re} s>1 / 2$ it is given by a Dirichlet series with positive coefficients. The Ikehara Tauberian theorem (see, e.g., [27], Ch. V, §17, or [17], Ch. XV, §2, 3) implies that

$$
\sum_{(c, d) \in \mathcal{B}, c<X} \frac{1}{c} \sim \frac{2 q \lambda^{2}}{\pi^{2}(q-2)} X \quad(X \rightarrow \infty) .
$$

By partial summation this implies the statements in the lemma.

5.9. Taylor expansion. For $\operatorname{Re} u \geq \sigma, \operatorname{Re} u \notin \mathbb{Z}$,

$$
\Phi_{\nu}^{\mu}(r, u)=\sum_{k=0}^{l-1} \frac{r^{k}}{k !}\left(\frac{\pi i}{2 q}\right)^{k} \Delta_{k}(\nu, \mu, 1+2 u-k)+\mathcal{O}\left(r^{2 \sigma-1}\right) \quad(r \downarrow 0) .
$$

The constant implied in $\mathcal{O}$ does not depend on the choice of $u$, provided $\operatorname{Re} u \geq \sigma$.

This corresponds to Lemma 3.6 in [5], and is obtained in the same way: The terms with $c \geq r^{-1}$ in the series for $\Phi_{\nu}^{\mu}$ give a contribution $\mathcal{O}\left(r^{2 \operatorname{Re} u-1}\right)=\mathcal{O}\left(r^{2 \sigma-1}\right)$. For the other terms consider a Taylor expansion of $e^{\pi i r T(c, d) /(2 q)}$ up to the term with $r^{l-1}$. The remainder terms give a contribution estimated by

$$
r^{l} \sum_{(c, d) \in \mathcal{B}, c<1 / r} c^{l-2 \sigma-1}=\mathcal{O}\left(r^{2 \sigma-1}\right) .
$$

In the other terms we go over from $c<1 / r$ to all $c$; the difference is again $\mathcal{O}\left(r^{2 \sigma-1}\right)$.

5.10. The final sum. We insert the expansion in (5.2) into

$$
e^{-\pi i r / 2} \varphi_{\nu}^{\mu}(r, s)=\sum_{p=0}^{\infty} \alpha_{p}(n, m, s) \Phi_{\nu}^{\mu}(r, s+p) .
$$


As $\sum_{p=0}^{\infty} \alpha_{p}(n, m, s)=\mathcal{O}(1)(r \downarrow 0)$, the remainder term contributes $\mathcal{O}\left(r^{2 \sigma-1}\right)$. For each $k \in[0, l-1]$, and $s$ still fixed, the series

$$
\sum_{p=0}^{\infty} \Delta_{k}(\nu, \mu, 1+2 s+2 p-k) \alpha_{p}(n, m, s)
$$

converges absolutely, uniformly for $r$ in a neighborhood of 0 . It defines a holomorphic function of $r$. We replace it by its expansion up to $\mathcal{O}\left(r^{l}\right)$, and get

$$
\begin{aligned}
e^{-\pi i r / 2} \varphi_{\nu}^{\mu}(r, s) \\
=\sum_{k=0}^{l-1} \frac{r^{k}}{k !}\left(\frac{\pi i}{2 q}\right)^{k} \sum_{p=0}^{\infty}\left(\frac{-4 \pi^{2}}{\lambda^{2}}\right)^{p} \frac{(m n)^{p}}{p !(1+2 s)_{p}} \Delta_{k}(\nu, \mu, 1+2 s+2 p-k) \\
\quad+\mathcal{O}\left(r^{2 \sigma-1}\right) \\
=\sum_{h=0}^{l-1} r^{h} \sum_{p=0}^{\infty} \sum_{k=0}^{h} \beta_{p, k}^{h}(\nu, \mu, s) \Delta_{k}(\nu, \mu, 1+2 s+2 p-k)+\mathcal{O}\left(r^{2 \sigma-1}\right),
\end{aligned}
$$

with $\beta_{p, k}^{h}$ as in Proposition 5.6.

6. Meromorphic continuation. It is well known that Eisenstein and Poincaré series have a meromorphic continuation in the spectral parameter $s$, and satisfy a functional equation. The case of weight 0 may be found in $[22]$. In $[2,3,4]$ the meromorphic continuation in $(r, s)$ jointly has been studied. These papers treat the modular case, $q=3$. Extension to all $G_{q}$ is straightforward for the results in [2,3], and for part of the results in [4]. Here we need only results that extend easily from $G_{3}$ to $G_{q}$. Theorem 10.2.1 in [9] gives these results in a more general context. (To obtain the results in 6.1 one takes the cell of continuation $J$ equal to the null cell $J(0)$.)

Our aim is to obtain the meromorphic continuation of the $\Delta_{k}$, and information concerning the right-most singularity.

6.1. Meromorphic continuation in two parameters. Proposition 2.19 in [3], generalized to $G_{q}$, gives:

Let $\mu \in \mathbb{Z}$. There are a neighborhood $U$ of 0 in $\mathbb{C}$, containing $\left(\frac{-4 q}{q-2}, \frac{4 q}{q-2}\right)$, and a meromorphic family $E^{\nu}$ of automorphic forms on $U \times \mathbb{C}$ that is uniquely determined by the following conditions:

(i) For each $(r, s) \in U \times \mathbb{C}$ at which $E^{\mu}$ is holomorphic it is an automorphic form of weight $r$, with eigenvalue $1 / 4-s^{2}$ and multiplier system $v_{r}$.

(ii) There are meromorphic functions $C_{\nu}^{\mu}$ on $U \times \mathbb{C}$ such that

$$
E^{\mu}(r, s ; \lambda z)=\mu^{m}(r, s ; z)+C_{0}^{\mu}(r, s) \mu^{n_{0}}(r,-s ; z)+\sum_{\nu \neq 0} C_{\nu}^{\mu}(r, s) \omega^{n}(r, s ; z)
$$


as an identity of meromorphic functions. We understand the quantities $m=\mu+(q-2) r /(4 q)$ and $n=\nu+(q-2) r /(4 q)$ to vary with $r$; by $n_{0}$ we mean $(q-2) r /(4 q)$.

The characterization of $E^{\mu}$ by the form of its Fourier series expansion implies various functional equations. Here we shall use the following ones: $E^{0}(r,-s)=C_{0}^{0}(r,-s) E^{0}(r, s), E^{0}(r, s ;-\bar{z})=E^{0}(-r, s ; z)$, and $C_{0}^{0}(r,-s) C_{0}^{0}(r, s)=1$.

A holomorphic family of automorphic forms is a $C^{\infty}$-function in $(r, s, z)$ that is holomorphic in $(r, s)$ if we leave $z$ fixed, and that is an automorphic form in $z$ if we leave $(r, s)$ fixed. A meromorphic family is understood to be locally in $(r, s)$ of the form $\frac{1}{\psi(r, s)} f(r, s, z)$, with $f$ a holomorphic family, and $\psi$ a holomorphic function that is not identically zero. So all meromorphic functions $(r, s) \mapsto E^{\mu}(r, s ; z)$ have common denominators.

Generalization of Proposition 2.23 in [3], or application of part (ii) of Theorem 10.2.1 in [9], implies:

The family $s \mapsto E^{\mu}(0, s)$ exists as a meromorphic family of automorphic forms of weight 0 . It is holomorphic on $\operatorname{Re} s>1 / 2$, and is given by the Poincaré series $p^{\mu}(0, s)$ for $\operatorname{Re} s>1 / 2$.

The existence of the restriction $s \mapsto E^{\mu}(0, s)$ is not trivial, as $E^{\mu}$ might have had a singularity along the line $r=0$. The holomorphy at $s=s_{0}$ of this restriction need not imply the holomorphy of $E^{\mu}$ at $\left(0, s_{0}\right)$; see 6.7 .

6.2. Fourier coefficients. The $C_{\nu}^{\mu}$ are meromorphic on $U \times \mathbb{C}$, the restrictions $s \mapsto C_{\nu}^{\mu}(0, s)$ exist, and coincide with $c_{\nu}^{\mu}(0, s)$ for $\operatorname{Re} s>1 / 2$. It will turn out to be useful not to work with $C_{\nu}^{\mu}$, but with $\Psi_{\nu}^{\mu}$, defined by $C_{\nu}^{\mu}(r, s)=\lambda^{-1-2 s} g_{\nu}(r, s) \Psi_{\nu}^{\mu}(r, s)$, with

$$
\begin{aligned}
& g_{0}(r, s)=\frac{\pi 2^{1-2 s} \Gamma(2 s)}{\Gamma(1 / 2+s+r / 2) \Gamma(1 / 2+s-r / 2)}, \\
& g_{\nu}(r, s)=\frac{\pi^{s+1 / 2}}{\Gamma(1 / 2+s \pm r / 2)}\left( \pm \nu \pm \frac{q-2}{4 q} r\right)^{s-1 / 2} \quad \text { if } \pm \nu>0 .
\end{aligned}
$$

Each $\Psi_{\nu}^{\mu}$ has a meromorphic restriction to the line $r=0$; on $\operatorname{Re} s>1 / 2$ it is holomorphic with value $\varphi_{\nu}^{\mu}(0, s)$.

6.3. Derivatives. For each integral $h \geq 0$ the function

$$
\psi_{\nu}^{\mu}(h ; s)=\frac{1}{h !} \partial_{r}^{h} \Psi_{\nu}^{\mu}(0, s)
$$

is meromorphic on $\mathbb{C}$. Of course, $\psi_{\nu}^{\mu}(0 ; s)$ is just the restriction $s \mapsto \Psi_{\nu}^{\mu}(0, s)$.

6.4. Poincaré series of positive weight. Let $U^{+}=\{r \in U: \operatorname{Re} u>0\}$. On $U^{+} \times \mathbb{C}$ there is no reason not to use the basis $\mu^{n_{0}}(r, s), \omega^{n_{0}}(r, s)$ for the Fourier terms of order $n_{0}=(q-2) r /(4 q)$. In Proposition 2.20 of [3] the 
$E_{\nu}^{\mu}$ are combined to form meromorphic families $P^{\mu}$ on $U^{+} \times \mathbb{C}$ that have a Fourier expansion with a simple form with respect to the latter basis. (One may also apply Theorem 10.2.1 in [9] with $J$ equal to a minimal cell of continuation, and consult the discussion in 10.3.2 of [9].) To make this explicit we need

$$
v(r, s)=\left(\frac{q-2}{q} \pi r\right)^{s+1 / 2} \frac{\Gamma(-2 s)}{\Gamma(1 / 2-s-r / 2)},
$$

for which $\omega^{n_{0}}(r, s)=v(r, s) \mu^{n_{0}}(r, s)+v(r,-s) \mu^{n_{0}}(r,-s)$. It turns out that

$$
P^{\mu}(r, s)=E^{\mu}(r, s)+\frac{v(r, s) C_{0}^{\mu}(r, s)}{v(r,-s)-v(r, s) C_{0}^{0}(r, s)} E^{0}(r, s) .
$$

It has a Fourier expansion

$$
P^{\mu}(r, s)=\mu^{m}(r, s)+\sum_{\nu} D_{\nu}^{\mu}(r, s) \omega^{n}(r, s),
$$

with $D_{\nu}^{\mu}$ the meromorphic function on $U^{+} \times \mathbb{C}$ given by

$$
\begin{aligned}
& D_{0}^{\mu}(r, s)=C_{0}^{\mu}(r, s) /\left(v(r,-s)-v(r, s) C_{0}^{0}(r, s)\right), \\
& D_{\nu}^{\mu}(r, s)=C_{\nu}^{\mu}(r, s)+\frac{v(r, s) C_{0}^{\mu}(r, s) C_{\nu}^{0}(r, s)}{v(r,-s)-v(r, s) C_{0}^{0}(r, s)} \quad \text { if } \nu \neq 0 .
\end{aligned}
$$

Proposition 2.23 in [5] can be generalized to state that $P^{\mu}$ is holomorphic at $(r, s)$ if $0<r<(q-2) /(4 q)$ and $\operatorname{Re} s>1 / 2$, with value $p^{m}(r, s)$. (Remember the convention $m=\mu+(q-2) r /(4 q)$.) Hence for these values of $(r, s)$ the $D_{\nu}^{\mu}(r, s)$ are given by the $c_{n}^{m}(r, s)$. (In [9] I call a family like $P^{\mu}$ a family on a restricted parameter space; see Proposition 10.2.12 for the holomorphy.)

This makes it possible to express the $\Psi_{\nu}^{\mu}$ in terms of the $\varphi_{\nu}^{\mu}$. We do not carry this out completely, but are content with inverting the relation between the $C$ 's and the $D$ 's:

$$
\begin{aligned}
& C_{0}^{\mu}(r, s)=\frac{v(r,-s) D_{0}^{\mu}(r, s)}{1+v(r, s) D_{0}^{0}(r, s)} \\
& C_{\nu}^{\mu}(r, s)=D_{\nu}^{\mu}(r, s)-\frac{v(r, s) D_{0}^{\mu}(r, s) D_{\nu}^{0}(r, s)}{1+v(r, s) D_{0}^{0}(r, s)} \quad \text { if } \nu \neq 0 .
\end{aligned}
$$

6.5. Behavior for $r \downarrow 0$. Let us take $l \in \mathbb{N}, l \geq 2$. The restriction $s \mapsto \Psi_{\nu}^{\mu}(0, s)$ is meromorphic, hence the strip $\{s \in \mathbb{C}: l / 2<\operatorname{Re} s<(l+1) / 2\}$ contains an open dense subset of points $s$ such that $\Psi_{\nu}^{\mu}$ is holomorphic at $(0, s)$.

Let us fix such an $s$, and put $\sigma=\operatorname{Re} s$. We want to consider the behavior for $r \downarrow 0$ of $\Psi_{\nu}^{\mu}(r, s)=\lambda^{-1-2 s} g_{\nu}(r, s)^{-1} C_{\nu}^{\mu}(r, s)$. We express $C_{\nu}^{\mu}$ in the $D$ 's, which are equal to the corresponding $c_{n}^{m}(r, s)=G_{r}(n, m, s) \varphi_{\nu}^{\mu}(r, s)$ for the 
current $(r, s)$. The $c$ 's we express in the $\varphi_{\nu}^{\mu}$, for which Proposition 5.6 gives a Taylor expansion in $r$.

$$
\begin{aligned}
v(r, s) D_{0}^{\mu}(r, s)= & e^{-\pi i r / 2} \lambda^{-1-2 s}\left(\frac{q-2}{4 q} \pi r\right)^{2 s} \\
& \times \frac{2^{2 s+1} \Gamma(-2 s)}{\Gamma(1 / 2-s-r / 2) \Gamma(1 / 2+s+r / 2)} \varphi_{0}^{\mu}(r, s) \\
= & \mathcal{O}\left(r^{2 \sigma}\right), \\
& \times \frac{\pi^{s+1 / 2}}{\Gamma(1 / 2+s+(r / 2) \operatorname{sign} \nu)} \varphi_{\nu}^{0}(r, s) \\
D_{\nu}^{0}(r, s)= & e^{-\pi i r / 2} \lambda^{-1-2 s}\left|\nu+\frac{q-2}{4 q} r\right|^{s-1 / 2} \\
C_{0}^{\mu}(r, s)= & v(r,-s) D_{0}^{\mu}(r, s)\left(1+\mathcal{O}\left(r^{2 \sigma}\right)\right), \\
C_{\nu}^{\mu}(r, s)= & D_{\nu}^{\mu}(r, s)+\mathcal{O}\left(r^{2 \sigma}\right) \quad(\text { with } \nu \neq 0), \\
\Psi_{0}^{\mu}(r, s)= & \lambda^{1+2 s} g_{0}(r, s)^{-1} v(r,-s) D_{0}^{\mu}(r, s)\left(1+\mathcal{O}\left(r^{2 \sigma}\right)\right) \\
= & e^{-\pi i r / 2} \varphi_{0}^{\mu}(r, s)+\mathcal{O}\left(r^{2 \sigma}\right), \\
\Psi_{\nu}^{\mu}(r, s)= & \lambda^{1+2 s} g_{\nu}(r, s)^{-1} D_{\nu}^{\mu}(r, s)+\mathcal{O}\left(r^{2 \sigma}\right) \\
= & e^{-\pi i r / 2} \varphi_{\nu}^{\mu}(r, s)+\mathcal{O}\left(r^{2 \sigma}\right) \quad(\text { with } \nu \neq 0) .
\end{aligned}
$$

$\Psi_{\nu}^{\mu}$ is holomorphic at $(0, s)$, and the $\psi_{\nu}^{\mu}(h ; s)$ are the Taylor coefficients at 0 of the holomorphic function $r \mapsto \Psi_{\nu}^{\mu}(r, s)$. We find the first $l$ of them in Proposition 5.6. This can be done for a dense set of points $s$ in the strip $l / 2<\operatorname{Re} s<(l+1) / 2$. We obtain an identity between meromorphic functions that extends to the region on which both sides are defined:

6.6. Lemma. Let $\mu, \nu \in \mathbb{Z}$, and $h \in \mathbb{N} \cup\{0\}$. The meromorphic function $\psi_{\nu}^{\mu}(h)$ is holomorphic for $\operatorname{Re} s>(h+1) / 2$, and satisfies on this region

$$
\psi_{\nu}^{\mu}(h ; s)=\sum_{p=0}^{\infty} \sum_{k=0}^{h} \beta_{p, k}^{h}(\nu, \mu, s) \Delta_{k}(\nu, \mu, 1+2 s+2 p-k),
$$

with $\beta_{p, k}^{h}$ as in Proposition 5.6.

6.7. Singularities at half-integral points. Now we turn to the generalization of Proposition 7.19 in [3]. It describes the behavior of the $E^{\mu}$ at points $(0, s)$ with $\operatorname{Re} s>1 / 2$.

Singularities of such Poincaré families at points $(0, s)$ with $\operatorname{Re} s \geq 0$ generally arise from two causes. The first cause are spectral features: if the Laplacian on $\mathfrak{H}$ has square integrable eigenfunctions on $G_{q} \backslash \mathfrak{H}$ with eigenvalue $1 / 4-s^{2}$ with non-vanishing Fourier coefficient of order $\mu$, then $E^{\mu}$ 
may have a singularity at $(0, s)$. In weight zero all such eigenvalues are in $[0, \infty)$; so for $\operatorname{Re} s>1 / 2$ this possibility is of no concern. The other main cause for singularities is more trivial. The families $E^{\mu}$ are determined by a prescribed form of the Fourier coefficients with respect to a fixed basis of the space of possible Fourier terms. If the functions used fail to be a basis at some point, trouble is to be expected. In the present case this occurs at points $(0, l / 2), l \in \mathbb{N}$, due to the singularity of $\mu^{n_{0}}(r,-s)$ at these points. If we look at the restriction to the line $r=0$ this is not visible, as $\mu^{n_{0}}(0,-s ; z)=y^{-s+1 / 2}$. To describe the resulting singularity one goes over to another basis of the Fourier terms of order $n_{0}$. Take a meromorphic function $w_{l}$ such that $\mu^{n_{0}}(r, s)$ and ${ }^{l} \nu(r, s)=\mu^{n_{0}}(r,-s)+w_{l}(r, s) \mu^{n_{0}}(r, s)$ form a basis suitable for all points near $(0, l / 2)$. For all other Fourier terms the old basis is all right. Define linear combinations ${ }^{l} E^{\mu}$ of the $E$ 's with a simple Fourier expansion with respect to the new basis. These ${ }^{l} E^{\mu}$ behave nicely at $(0, l / 2)$. In $[3]$ I used

$$
w_{l}(r, s)=\left(\frac{\pi(q-2) r}{q}\right)^{l} \frac{\Gamma(-2 s) \Gamma(1 / 2+s-r / 2)}{\Gamma(2 s) \Gamma(1 / 2-s-r / 2)} ;
$$

at present I prefer

$$
\left(\frac{\pi(q-2) r}{q}\right)^{l} \frac{\Gamma(-2 s)}{\Gamma(2 s)}\left(\frac{1}{2}-s-\frac{r}{2}\right)_{l} .
$$

Both can be used to get the following generalization of Proposition 7.19 in [3]:

Let $\mu \in \mathbb{Z}, s_{0} \in \mathbb{C}, \operatorname{Re} s_{0}>1 / 2$.

(i) If $s_{0} \notin(1 / 2) \mathbb{N}$, then $E^{\mu}$ is holomorphic at $\left(0, s_{0}\right)$, with value given by the Poincaré series $p^{\mu}\left(0, s_{0}\right)$.

(ii) Let $s_{0}=l / 2, l \in \mathbb{N}, l \geq 2$. Put

$$
{ }^{l} E^{\mu}(r, s)=E^{\mu}(r, s)+\frac{w_{l}(r, s) C_{0}^{\mu}(r, s)}{1-w_{l}(r, s) C_{0}^{0}(r, s)} E^{0}(r, s) .
$$

Then ${ }^{l} E^{\mu}$ is holomorphic at $\left(0, s_{0}\right)$, with value given by $p^{\mu}\left(s_{0}\right)$.

This is a special case of Propositions 12.4.2 and 12.4.3 in [9].

The fact that ${ }^{l} E^{\mu}$ has value $p^{\mu}(l / 2)$ at $(0, l / 2)$ is not stated explicitly in [3]; it follows from the fact that $E^{\mu}$ and ${ }^{l} E^{\mu}$ have the same restriction to the line $r=0$.

This implies that the $C_{\nu}^{\mu}$ are holomorphic at $(0, s)$ for $\operatorname{Re} s>1 / 2, s \notin$ $(1 / 2) \mathbb{Z}$.

Near points $(0, l / 2), l \geq 2, l \in \mathbb{N}$, we have

$$
{ }^{l} E^{\mu}(\lambda z)=\mu^{m}(r, s)+{ }^{l} C_{0}^{\mu}(r, s){ }^{l} \nu(r, s)+\sum_{\nu \neq 0}{ }^{l} C_{\nu}^{\mu}(r, s) \omega^{n}(r, s),
$$




$$
\begin{aligned}
{ }^{l} C_{\nu}^{\mu}(r, s) & =C_{\nu}^{\mu}(r, s)+\frac{w_{l}(r, s) C_{0}^{\mu}(r, s) C_{\nu}^{0}(r, s)}{1-w_{l}(r, s) C_{0}^{0}(r, s)}, \\
C_{\nu}^{\mu}(r, s) & ={ }^{l} C_{\nu}^{\mu}(r, s)-\frac{w_{l}(r, s){ }^{l} C_{0}^{\mu}(r, s){ }^{l} C_{\nu}^{0}(r, s)}{1+w_{l}(r, s){ }^{l} C_{0}^{0}(r, s)} .
\end{aligned}
$$

The ${ }^{l} C_{\nu}^{\mu}$ are holomorphic at $(0, l / 2)$, with value $c_{\nu}^{\mu}(0, l / 2)$.

6.8. Lemma. Let $h \in \mathbb{N} \cup\{0\}$, and $\mu, \nu \in \mathbb{Z}$. All singularities of $\psi_{\nu}^{\mu}(h)$ in the region $\operatorname{Re} s>1 / 2$ occur at half-integral points.

(i) There exists a number $\sigma_{0} \in(0,1 / 2)$ such that all $\psi_{\nu}^{\mu}(0)$ are holomorphic on the region $\operatorname{Re} s>\sigma_{0}$, with the exception of a first order pole at $s=1 / 2$ in the case $\mu=\nu=0$, with residue $q \lambda^{2} \pi^{-2}(q-2)^{-1}$.

(ii) $\psi_{\nu}^{\mu}(1)$ is holomorphic on $\operatorname{Re} s>1 / 2$.

(iii) If $h$ is odd, $h \geq 3$, then $\psi_{\nu}^{\mu}(h)$ is holomorphic on $\operatorname{Re} s>(h-1) / 2$.

(iv) Let $h$ be even, $h \geq 2$. If the number

$$
\frac{(-1)^{h / 2}}{h !}\left(\frac{\pi(q-2)}{2 \lambda q}\right)^{h} \lambda^{-1} \varphi_{0}^{\mu}(0, h / 2) \varphi_{0}^{\nu}(0, h / 2)
$$

vanishes, then $\psi_{\nu}^{\mu}(h)$ is holomorphic at $s=h / 2$, otherwise $\psi_{\nu}^{\mu}(h)$ has a first order pole at $s=h / 2$, with this number as the residue. There are no other singularities of $\psi_{\nu}^{\mu}(h)$ in the region $\operatorname{Re} s>(h-1) / 2$.

The proof will take the remainder of this section. The case $h=0$ is just spectral theory of automorphic forms. The other cases are based on (6.3). From the results stated above it follows that the only singularities in $\operatorname{Re} s>$ $1 / 2$ occur at points $l / 2$ with $l \in \mathbb{N}, l \geq 2$.

6.9. Case $h=0$. The $\psi_{\nu}^{\mu}(0)$ are, up to some explicit factors, the Fourier coefficients of Eisenstein and Poincaré series in weight 0. See, e.g., [16], Ch. VI, Theorems 11.6 and 11.8 on pp. 128-130, claim 9.2 on p. 71 , claim 9.6 on p. 78 , Theorem 11.11 on p. 140, and also Ch. VII, Theorem 5.3(i), (ii) and Proposition 5.5(i), (ii) on p. 258. Choose the number $\sigma_{0}$ in such a way that there are no automorphic eigenvalues in the interval $\left(0,1 / 4-\sigma_{0}^{2}\right)$.

The holomorphy of $p^{\mu}$ at $s=1 / 2$, for $\mu \neq 0$, is a consequence of Proposition 11.3.9(iii) in [9].

6.10. Case $h \geq 1$. In the lemma we fix $h$, and consider the $l \geq 2$, $l \in \mathbb{N}$, at which $\psi_{\nu}^{\mu}(h)$ is singular. In the proof it is more convenient to fix $l \in \mathbb{N}, l \geq 2$, and to consider the $h$ for which $\psi_{\nu}^{\mu}(h)$ is holomorphic at $l / 2$. This means that we have to prove that $\psi_{\nu}^{\mu}(h)$ is holomorphic at $s=l / 2$ for $1 \leq h \leq l-1$, and also for $h=l$ if $l$ is odd. For even $l$ we have to determine the value at $s=l / 2$ of $s \mapsto(s-l / 2) \psi_{\nu}^{\mu}(l)$.

We have $\Psi_{\nu}^{\mu}(r, s)=\sum_{h=0}^{\infty} \psi_{\nu}^{\mu}(h ; s) r^{h}$ for $r$ near 0 , and $s$ near $l / 2, s \neq$ $l / 2$. The function $C_{\nu}^{\mu}(r, s)=\lambda^{-1-2 s} g_{\nu}(r, s) \Psi_{\nu}^{\mu}(r, s)$ has a similar expansion 
$C_{\nu}^{\mu}(r, s)=\sum_{h=0}^{\infty} B_{\nu}^{\mu}(h ; s) r^{h}$. As $g_{\nu}$ is holomorphic and non-zero at $(0, l / 2)$, it suffices to prove for the $B_{\nu}^{\mu}$ :

( $\alpha) B_{\nu}^{\mu}(h)$ is holomorphic at $s=l / 2$ for $1 \leq h \leq l-1$.

( $\beta) s \mapsto(s-l / 2) B_{\nu}^{\mu}(l)$ is holomorphic at $s=l / 2$, with value

$$
\varrho_{\nu}^{\mu}(l)= \begin{cases}\frac{(-1)^{l / 2}}{l ! \lambda^{2}}\left(\frac{\pi(q-2)}{2 q \lambda^{2}}\right)^{l} g_{\nu}(0, l / 2) \varphi_{0}^{\mu}(0, l / 2) \varphi_{0}^{\nu}(0, l / 2) & \text { if } l \text { is even } \\ 0 & \text { if } l \text { is odd }\end{cases}
$$

We treat three cases separately: $\mu$ and $\nu$ both zero, one of them zero, and both non-zero. The first two cases have been considered in [5]. There I tried to get information on the order of all poles at points between $1 / 2$ and $h / 2$; that made the treatment messy. I repeat the reasoning here, but restrict myself to the right-most singularity.

6.11. Case $\mu=\nu=0$. From the functional equation $E^{0}(r, s ;-\bar{z})=$ $E^{0}(-r, s ; z)$ it follows that $C_{0}^{0}(-r, s)=C_{0}^{0}(r, s)$. Hence the $B_{0}^{0}(2 k+1)$ vanish, and we can restrict the discussion to even $h$.

There are meromorphic functions $A_{2 k}$ and holomorphic functions ${ }^{l} A_{h}$ on a neighborhood of $s=l / 2$ such that

$$
C_{0}^{0}(r, s)^{-1}=\sum_{k=0}^{\infty} A_{2 k}(s) r^{2 k}, \quad{ }^{l} C_{0}^{0}(r, s)^{-1}=\sum_{h=0}^{\infty}{ }^{l} A_{h}(s) r^{h} .
$$

To see that ${ }^{l} C_{0}^{0}(0, l / 2) \neq 0$, use the fact that it is equal to $c_{0}^{0}(0, l / 2)$, and that $\varphi_{0}^{0}(0, l / 2)>0$.

The $r$-expansion of $w_{l}$ starts with

$$
w_{l}(r, s)=r^{l}\left(\frac{\pi(q-2)}{q}\right)^{l}\left(\frac{1}{2}-s\right)_{l} \frac{\Gamma(-2 s)}{\Gamma(2 s)}+\ldots ;
$$

I use the version of $w_{l}$ given in (6.2).

As $C_{0}^{0}(r, s)^{-1}={ }^{l} C_{0}^{0}(r, s)^{-1}+w_{l}(r, s)$, we conclude that the $A_{2 k}$ with $2 k<l$ are holomorphic at $s=l / 2$. If $l$ is even, the singular part of $A_{l}$ at $s=l / 2$ is given by the first term in the expansion of $w_{l}$. This gives, for even $l$,

$$
A_{l}(s)=\frac{1}{s-l / 2}\left(\frac{\pi(q-2)}{2 q}\right)^{l} \frac{(-1)^{1+l / 2}}{g_{0}(0, l / 2) l !}+(\text { holomorphic at } l / 2) .
$$

The relation between the $A$ 's and the $B_{0}^{0}$ 's implies that $B_{0}^{0}(2 k)$ is holomorphic at $s=l / 2$ for $2 k<l$, and that for even $l$, modulo holomorphic functions at $s=l / 2$ : 


$$
\begin{aligned}
B_{0}^{0}(l ; s) & \equiv-B_{0}^{0}(0 ; s)^{2} A_{l}(s) \\
& \equiv-\lambda^{-2-2 l} g_{0}(0, l / 2)^{2} \varphi_{0}^{0}(0, l / 2)^{2} \frac{(-1)^{1+l / 2}}{g_{0}(0, l / 2) l !}\left(\frac{\pi(q-2)}{2 q}\right)^{l} \frac{1}{s-l / 2} \\
& =\varrho_{0}^{0}(l) \frac{1}{s-l / 2} .
\end{aligned}
$$

This gives $(\alpha)$ and $(\beta)$.

6.12. Case $\mu \nu=0$, but $\mu \neq 0$ or $\nu \neq 0$. We consider the quotient

$$
Q(r, s)=C_{\nu}^{\mu}(r, s) / C_{0}^{0}(r, s)={ }^{l} C_{\nu}^{\mu}(r, s) /{ }^{l} C_{0}^{0}(r, s) .
$$

As ${ }^{l} C_{0}^{0}(0, l / 2)$ is non-zero, the function $Q$ is holomorphic at $(0, l / 2)$, with value

$$
Q(0, l / 2)=\frac{c_{\nu}^{\mu}(0, l / 2)}{c_{0}^{0}(0, l / 2)}=\frac{g_{\nu}(0, l / 2)}{g_{0}(0, l / 2)} \cdot \frac{\varphi_{\nu}^{\mu}(0, l / 2)}{\varphi_{0}^{0}(0, l / 2)} .
$$

With $A_{2 k}$ as above this gives

$$
\begin{aligned}
Q(r, s) & =\sum_{h=0}^{\infty} r^{h} \sum_{0 \leq k \leq h / 2} A_{2 k}(s) B_{\nu}^{\mu}(h-2 k ; s), \\
B_{\nu}^{\mu}(h ; s) & =\left.B_{0}^{0}(0 ; s) \frac{1}{h !} \partial_{r}^{h} Q(r, s)\right|_{r=0}-\sum_{1 \leq k \leq h / 2} B_{0}^{0}(0 ; s) A_{2 k}(s) B_{\nu}^{\mu}(h-2 k ; s) .
\end{aligned}
$$

We already know that the function $B_{\nu}^{\mu}(0)$ is holomorphic at $s=l / 2$ with value $\lambda^{-1-l} g_{\nu}(0, l / 2) \varphi_{\nu}^{\mu}(0, l / 2)$. Inductively we get the holomorphy of $B_{\nu}^{\mu}(h)$ at $s=l / 2$ for $h<l$, and for $h=l$ if $l$ is odd. For even $l$ we get, modulo holomorphic functions at $s=l / 2$,

$$
\begin{aligned}
B_{n}^{\mu}(l ; s) \equiv & -B_{0}^{0}(0 ; s) A_{l}(s) B_{\nu}^{\mu}(0 ; s) \\
\equiv & \frac{1}{s-l / 2}(-1) \lambda^{-1-l} g_{0}(0, l / 2) \varphi_{0}^{0}(0, l / 2) \frac{(-1)^{1+l / 2}}{g_{0}(0, l / 2) l !}\left(\frac{\pi(q-2)}{2 q}\right)^{l} \\
& \times \lambda^{-1-l} g_{\nu}(0,1 / 2) \varphi_{\nu}^{\mu}(0, l / 2) \\
= & \varrho_{\nu}^{\mu}(l) \frac{1}{s-l / 2} .
\end{aligned}
$$

Use $\varphi_{\nu}^{\mu}(0, s)=\varphi_{\mu}^{\nu}(0, s)$ (see 5.4) to conclude that $\varphi_{0}^{0} \varphi_{\nu}^{\mu}=\varphi_{0}^{\mu} \varphi_{0}^{\nu}$ in the case $\mu=0$.

6.13. Case $\mu \nu \neq 0$. In (6.3) we have expressed $C_{\nu}^{\mu}$ in the ${ }^{l} C_{\text {. }}$. As ${ }^{l} C_{\nu}^{\mu}$ is holomorphic at $(0, l / 2)$, we need look only at the correction term given by

$$
R(r, s)={ }^{l} C_{\nu}^{\mu}(r, s)-C_{\nu}^{\mu}(r, s)=w_{l}(r, s) \frac{{ }^{l} C_{0}^{\mu}(r, s){ }^{l} C_{\nu}^{0}(r, s)}{1+w_{l}(r, s){ }^{l} C_{0}^{0}(r, s)} .
$$


Now, $R(r, s) /\left(w_{l}(r, s) C_{0}^{0}(r, s)\right)={ }^{l} C_{0}^{\mu}(r, s)^{l} C_{\nu}^{0}(r, s) /{ }^{l} C_{0}^{0}(r, s)$ is holomorphic at $(r, s)=(0, l / 2)$ with value

$$
\frac{g_{\nu}(0, l / 2) \varphi_{0}^{\mu}(0, l / 2) \varphi_{\nu}^{0}(0, l / 2)}{\lambda^{1+l} \varphi_{0}^{0}(0, l / 2)} .
$$

The expansion of $w_{l}$ in (6.4) implies that the $r$-expansion of $R(r, s) / C_{0}^{0}(r, s)$ starts with $r^{l} f(s)+\ldots$, where, modulo terms holomorphic at $s=l / 2$,

$$
f(s) \equiv\left\{\begin{aligned}
\frac{1}{s-l / 2}\left(\frac{\pi(q-2)}{2 \lambda q}\right)^{l} \frac{(-1)^{1+l / 2}}{\lambda l !} \cdot \frac{g_{\nu}(0, l / 2) \varphi_{0}^{\mu}(0, l / 2) \varphi_{\nu}^{0}(0, l / 2)}{g_{0}(0, l / 2) \varphi_{0}^{0}(0, l / 2)} \\
\text { if } l \text { is even, } \\
\text { if } l \text { is odd. }
\end{aligned}\right.
$$

Hence the expansion of $R(r, s)$ starts with $r^{l} f(s) B_{0}^{0}(0 ; s)+\ldots$ This means that the $B_{\nu}^{\mu}(h)$ are holomorphic at $s=l / 2$ for $0 \leq h<l$, and that

$$
\begin{aligned}
\lim _{s \rightarrow l / 2}(s-l / 2) B_{\nu}^{\mu}(l ; s) & \\
\quad= & \lim _{s \rightarrow l / 2}(s-l / 2) f(s) B_{0}^{0}(0 ; s) \\
& = \begin{cases}\frac{(-1)^{l / 2}}{\lambda^{2} l !}\left(\frac{\pi(q-2)}{2 \lambda^{2} q}\right)^{l} g_{\nu}(0, l / 2) \varphi_{0}^{\mu}(0, l / 2) \varphi_{0}^{\nu}(0, l / 2) & \text { if } l \text { is even } \\
0 & \text { if } l \text { is odd } .\end{cases}
\end{aligned}
$$

7. Dirichlet series with Dedekind sums. The results in the previous sections lead to information concerning the right-most singularity of the Dirichlet series $\Delta_{h}(\nu, \mu, w)$ defined in (5.1). This will enable us to prove the distribution results in Propositions 1.4 and 1.5.

7.1. Proposition. Let $\mu, \nu, h \in \mathbb{Z}, h \geq 0$. The function $w \mapsto \Delta_{h}(\nu, \mu, w)$ has a meromorphic extension to $\mathbb{C}$.

(i) There exists $\varepsilon_{0} \in(0,1)$ such that

(a) $\Delta_{0}(\nu, \mu, w)$ is holomorphic on $\operatorname{Re} w>2-\varepsilon_{0}$ if $(\nu, \mu) \neq(0,0)$,

(b) $\Delta_{0}(0,0, w)$ is holomorphic on $\operatorname{Re} w>2-\varepsilon_{0}$, except for a first order pole at $w=2$ with residue $2 \lambda^{2} q /\left(\pi^{2}(q-2)\right)$.

(ii) $\Delta_{1}(\nu, \mu, w)$ is holomorphic on $\operatorname{Re} w>1$.

(iii) Let $h \geq 2$ be even. Then $\Delta_{h}(\nu, \mu, w)$ is holomorphic on $\operatorname{Re} w>0$, except possibly at $w=1$, and

$$
\lim _{w \rightarrow 1}(w-1) \Delta_{h}(\nu, \mu, w)=2(q-2)^{h} \lambda^{-h-1} \varphi_{0}^{\mu}(0, h / 2) \varphi_{0}^{\nu}(0, h / 2) .
$$

(iv) If $h \geq 3$ is odd, then $\Delta_{h}(\nu, \mu, w)$ is holomorphic on $\operatorname{Re} w>0$.

Proof. Central are the relation between the $\psi_{\nu}^{\mu}$ and the $\Delta_{k}(\mu, \nu, \cdot)$ in Lemma 6.6, and the properties of the $\psi_{\nu}^{\mu}$ given in Lemma 6.8. 
The meromorphic continuation is obtained by an induction procedure, based on the following reformulation of (6.1) in Proposition 6.6:

$$
\begin{aligned}
& \Delta_{h}(\nu, \mu, w) \\
= & h !\left(\frac{4 q}{2 \pi i}\right)^{h} \psi_{\nu}^{\mu}\left(h ; \frac{w-1+h}{2}\right) \\
& -h !\left(\frac{4 q}{2 \pi i}\right)^{h} \sum_{p} \sum_{k} \beta_{p, k}^{h}\left(\nu, \mu, \frac{w-1+h}{2}\right) \Delta_{k}(\nu, \mu, w+2 p+h-k),
\end{aligned}
$$

where $0 \leq k \leq h$, and $p \geq 1$ if $k=h, p \geq 0$ otherwise. Suppose $\Delta_{k}(\nu, \mu, w)$ has been meromorphically extended to $\operatorname{Re} w>2-m$. All terms in the right hand side of (7.1) are meromorphic on $\operatorname{Re} w>1-m$, but there are infinitely many of them. If $\operatorname{Re} w>1-m$, all but finitely many terms satisfy $\operatorname{Re}(w+2 p+h-k)>3$. On this region $\Delta_{k}$ is a bounded holomorphic function. The $\beta$-factors satisfy estimates ensuring that the corresponding infinite sum converges, and defines a meromorphic function. This gives the continuation to $\operatorname{Re} w>1-m$.

Consider the case $h=0$. Take $\varepsilon_{0}=2 \sigma_{0}$, with $\sigma_{0}$ as in Lemma 6.8. The term with $\psi_{\nu}^{\mu}(0)$ in (7.1) has the behavior described in (i)(a) and (b). For $\operatorname{Re} w>2-\varepsilon_{0}$ the remaining terms satisfy $\operatorname{Re}(w+2 p)>4-\varepsilon_{0}$, and hence define a holomorphic function.

For $h=1$ the term with $\psi_{\nu}^{\mu}(1)$ is holomorphic on $\operatorname{Re} w>1$. In the sum the terms with $p \geq 1$ satisfy $\operatorname{Re}(w+2 p+1-k)>3$. In the corresponding sum the $\Delta_{k}$ are bounded and the $\beta$-factors are holomorphic, hence the sum is holomorphic on $\operatorname{Re} w>1$. The remaining term with $p=k=0$ is also holomorphic on $\operatorname{Re} w>1$. This gives (ii).

To get (iii) and (iv), suppose that they have been proved for smaller values of $h$. It suffices to consider the various terms in (7.1) on the region $\operatorname{Re} w \geq \varepsilon$, for an arbitrary $\varepsilon \in(0,1)$. The subsum with $p \geq 1$ satisfies $\operatorname{Re}(w+2 p+h-k) \geq 2+\varepsilon$, hence it gives a holomorphic contribution. The other terms in the sum have $p=0$ and $k=0, \ldots, h-1$. For these $\operatorname{Re}(w+2 p+h-k) \geq 1+\varepsilon$. For $2 \leq k \leq h-1$ use the induction hypothesis. For $k=1$ use (ii). For $k=0$ we have $\operatorname{Re}(w+2 p+h-k)=\operatorname{Re}(w+h)>2$, hence (i) can be used. Finally, we consider $\psi_{\nu}^{\mu}\left(h ; \frac{w-1+h}{2}\right)$. Lemma 6.8 shows that it is holomorphic on $\operatorname{Re} w \geq \varepsilon$, except if $h$ is even. In that case there may be a singularity at $w=1$, with a residue that leads to the formula in (iii).

7.2. Proof of the distribution results. We shall use Proposition 7.1 to prove the distribution results for a suitable class of test functions. A density arguments will give Propositions 1.4 and 1.5 for all continuous functions. 
7.3. Some functionals. For continuous functions $f$ on $(\mathbb{R} \bmod \lambda \mathbb{Z})^{2} \times \mathbb{R}$ and $X>1$ define

$$
\begin{aligned}
\mu_{X}(f) & =\frac{1}{X} \sum_{(c, d) \in \mathcal{B}, c<X} \frac{1}{c} f\left(\frac{a}{c}, \frac{d}{c}, \frac{T(c, d)}{c}\right), \\
\mu(f) & =\frac{2 q}{\pi^{2}(q-2)} \int_{0}^{\lambda} \int_{0}^{\lambda} f(\xi, \eta, 0) d \xi d \eta ; \\
\nu_{X}(f) & =\frac{1}{X} \sum_{(c, d) \in \mathcal{B}, c<X}\left(\frac{T(c, d)}{c}\right)^{2} f\left(\frac{a}{c}, \frac{d}{c}, \frac{T(c, d)}{c}\right), \\
\nu(f) & =\sum_{\left(c_{1}, d_{1}\right) \in \mathcal{B}} \sum_{\left(c_{2}, d_{2}\right) \in \mathcal{B}} \frac{(q-2)^{2}}{\left(\lambda c_{1} c_{2}\right)^{3}} \sum_{ \pm} f\left(\frac{a_{1}}{c_{1}}, \frac{d_{2}}{c_{2}}, \frac{ \pm(q-2)}{\lambda c_{1} c_{2}}\right) .
\end{aligned}
$$

We use the convention that for each $(c, d) \in \mathcal{B}$ some $\left(\begin{array}{ll}a & b \\ c & d\end{array}\right) \in G_{q}$ has been chosen.

The second distribution result amounts to (7.3) below for all continuous $f$. We shall see in (7.6) that the first distribution result follows from (7.2) for all continuous $f$.

$$
\begin{aligned}
& \lim _{X \rightarrow \infty} \mu_{X}(f)=\mu(f), \\
& \lim _{X \rightarrow \infty} \nu_{X}(f)=\nu(f) .
\end{aligned}
$$

In 7.5 we prove (7.2) and (7.3) for the functions

$$
f_{\nu, \mu}^{h}(\xi, \eta, \sigma)=e^{2 \pi i(\mu \xi+\nu \eta) /(\lambda c)} \sigma^{h}, \quad \mu, \nu, h \in \mathbb{Z}, h \geq 0 .
$$

In 7.4 we extend these limit formulas to all continuous functions.

7.4. Extension. From (3.2) it follows that we can work in the space $Y$ of continuous functions on the compact set $(\mathbb{R} \bmod \lambda \mathbb{Z})^{2} \times\left[-\beta_{q}, \beta_{q}\right]$. Provide $Y$ with the supremum norm. The functions $f_{\nu, \mu}^{h}$ span a dense subspace $Y_{0}$. To extend the limit formulas from $Y_{0}$ to $Y$ we need $\left\|\mu_{X}\right\|=\mathcal{O}(1)(X \rightarrow \infty)$. This follows from $\left|\mu_{X}(f)\right| \leq\|f\| \mu_{X}\left(f_{0,0}^{0}\right)$, and $\lim _{X \rightarrow \infty} \mu_{X}\left(f_{0,0}^{0}\right)=\mu(1)$.

Proceed similarly for $\nu_{X}$.

7.5. Tauberian results. We use the Ikehara Tauberian theorem (see, e.g., [27], Ch. V, $\S 17$, or [17], Ch. XV, $\S 2,3)$. It allows us to conclude $\sum_{c<X} a(c) \sim$ $\varrho X(X \rightarrow \infty)$ from the fact that the Dirichlet series $\sum_{c \in \mathcal{C}} a(c) / c^{w}$ has a meromorphic extension to $\operatorname{Re} w>1-\varepsilon$, with a first order pole at $w=1$ with residue $\varrho$ as the only exception to holomorphy. The case that the extension is holomorphic at $w=1$ corresponds to $\varrho=0$. This can be applied directly if the $a(c)$ are non-negative. For complex $a(c)$ it holds if there are non-negative $b(c)$ with $a(c)=\mathcal{O}(b(c))$ such that the Dirichlet series $\sum_{c \in \mathcal{C}} b(c) / c^{w}$ satisfies similar assumptions, with non-zero residue at $w=1$. 
To get (7.2) for $f_{\nu, \mu}^{h}$ apply this with

$$
a(c)=c^{-1-h} \sum_{d,(c, d) \in \mathcal{B}} T(c, d)^{h} e^{2 \pi i(\mu a+\nu d) /(\lambda c)}, \quad b(c)=c^{-1} \#\{d:(c, d) \in \mathcal{B}\} .
$$

Together with Proposition 7.1 this gives

$$
\lim _{X \rightarrow \infty} \mu_{X}\left(f_{\nu, \mu}^{h}\right)= \begin{cases}2 \lambda^{2} q \pi^{-2}(q-2)^{-1} & \text { if } h=\mu=\nu=0 \\ 0 & \text { otherwise. }\end{cases}
$$

This turns out to be $\mu\left(f_{\nu, \mu}^{h}\right)$.

To get (7.3) for $f_{\nu, \mu}^{h}$ take

$$
\begin{aligned}
& a(c)=c^{-h-2} \sum_{d,(c, d) \in \mathcal{B}} T(c, d)^{h+2} e^{2 \pi i(\mu a+\nu d) /(\lambda c),} \\
& b(c)=c^{-h_{1}-2} \sum_{d,(c, d) \in \mathcal{B}} T(c, d)^{h_{1}+2},
\end{aligned}
$$

with $h_{1}=h$ if $h$ is even, and $h_{1}=h-1$ if $h$ is odd. The corresponding Dirichlet series are $\Delta_{h+2}(\nu, \mu, w)$ and $\Delta_{h_{1}+2}(0,0, w)$. We use the fact that $\varphi_{0}^{0}\left(0,1+h_{1} / 2\right)$ is positive. We get

$$
\begin{aligned}
\lim _{X \rightarrow \infty} \nu_{X}\left(f_{\nu, \mu}^{h}\right) & \\
& = \begin{cases}2(q-2)^{h+2} \lambda^{-h-3} \varphi_{0}^{\mu}(0, h / 2+1) \varphi_{0}^{\nu}(0, h / 2+1) & \text { if } h \text { is even } \\
0 & \text { if } h \text { is odd }\end{cases}
\end{aligned}
$$

To see that this equals $\nu\left(f_{\nu, \mu}^{h}\right)$ for even $h$, use $\varphi_{0}^{\mu}=\varphi_{\mu}^{0}$, and insert the Dirichlet series for the $\varphi$ 's.

7.6. Partial integration. Define $\alpha(X)=\sum_{(c, d) \in \mathcal{B}, c<X} c^{-1} f(a / c, b / d$, $T(c, d) / c)$ ), with $f$ continuous on $(\mathbb{R} \bmod \lambda \mathbb{Z})^{2} \times\left[-\beta_{q}, \beta_{q}\right]$. We have found $A \in \mathbb{C}$ such that $\alpha(X)=A X+o(X)(X \rightarrow \infty)$, and want to show that

$$
\int_{0}^{X} x d \alpha(x)=\frac{1}{2} A X^{2}+o\left(X^{2}\right) \quad(X \rightarrow \infty) .
$$

As

$$
\int_{0}^{X} x d \alpha(x)=X \alpha(X)-\int_{0}^{X} \alpha(x) d x,
$$

we have to show that

$$
\int_{0}^{X} \alpha(x) d x=\frac{1}{2} A X^{2}+o\left(X^{2}\right) .
$$

For $\varepsilon>0$ there exists $X_{\varepsilon}>1$ such that $|\alpha(x)-A x|<\varepsilon x$ for all $x>X_{\varepsilon}$. 
For $X>X_{\varepsilon}$ sufficiently large we have

$$
\left|\int_{0}^{X} \alpha(x) d x-\frac{A}{2} X^{2}\right| \leq(\text { constant })+\frac{\varepsilon}{2}\left(X^{2}-X_{\varepsilon}^{2}\right)<\varepsilon X .
$$

Thanks. I thank the copy editor of Acta Arithmetica for a number of corrections in this paper.

\section{References}

[1] M. Atiyah, The logarithm of the Dedekind $\eta$-function, Math. Ann. 278 (1987), 335-380.

[2] R. W. Bruggeman, Modular forms of varying weight. I, Math. Z. 190 (1985), 477-495.

[3] —, Modular forms of varying weight. II, ibid. 192 (1986), 297-328.

[4] —, Modular forms of varying weight. III, J. Reine Angew. Math. 371 (1986), 144-190.

[5] —, Eisenstein series and the distribution of Dedekind sums, Math. Z. 202 (1989), 181-198.

[6] —, On the distribution of Dedekind sums, in: Proc. Internat. Conf. Automorphic Functions and their Applications, Khabarovsk, 1988, N. Kuznetsov and V. Bykovsky (eds.), 82-89.

[7] -, Dedekind sums and Fourier coefficients of modular forms, J. Number Theory 36 (1990), 289-321.

[8] —, On the distribution of Dedekind sums, in: Contemp. Math. 166, Amer. Math. Soc., 1994, 197-210.

[9] —, Families of Automorphic Forms, Monographs Math. 88, Birkhäuser, 1994.

[10] R. Dedekind, Erläuterungen zu den Fragmenten XXVIII, in: B. Riemann, Gesammelte mathematische Werke und wissenschaftlicher Nachlass, H. Weber (ed.); reprint, Dover Publ., 1953, 466-478.

[11] L. J. Goldstein, Dedekind sums for a Fuchsian group, I, Nagoya Math. J. 50 (1973), 21-47.

[12] - Errata for Dedekind sums for a Fuchsian group, I, ibid. 53 (1974), 235-237.

[13] - Dedekind sums for a Fuchsian group, II, ibid. 53 (1974), 171-187.

[14] E. Hecke, Über die Bestimmung Dirichletscher Reihen durch ihre Funktionalgleichung, Math. Ann. 112 (1936), 664-699.

[15] - Dirichlet Series, Modular Functions and Quadratic Forms, lecture notes by H. Serbin, Institute of Adv. Study, Princeton, 1938.

[16] D. A. Hejhal, The Selberg Trace Formula for $\operatorname{PSL}(2, \mathbb{R})$, Vol. 2, Lecture Notes in Math. 1001, Springer, 1983.

[17] S. Lang, Algebraic Number Theory, Addison-Wesley, 1970.

[18] A. Leutbecher, Über die Heckeschen Gruppen $G(\lambda), I$, Abh. Math. Sem. Univ. Hamburg 31 (1967), 199-205.

[19] H. Maаß, Über eine neue Art von nichtanalytischen automorphen Funktionen und die Bestimmung Dirichletscher Reihen durch Funktionalgleichungen, Math. Ann. 121 (1949), 141-183.

[20] - Modular Functions of One Complex Variable, Tata Institute, Bombay, 1964; 2nd ed., Springer, 1983. 
[21] R. Matthes, The Eisenstein family for the modular group along closed geodesics, Math. Z., to appear.

[22] H. Neunhöffer, Über die analytische Fortsetzung von Poincaréreihen, Sitzungsber. Heidelb. Akad. Wiss. Math.-Natur. Kl., 2. Abhandlung, 1973, 33-90.

[23] H. Petersson, Zur analytischen Theorie der Grenzkreisgruppen, I, Math. Ann. 115 (1937), 23-67.

[24] H. Rademacher and E. Grosswald, Dedekind Sums, Math. Assoc. Amer., 1972.

[25] L. J. Slater, Confluent Hypergeometric Functions, Cambridge University Press, 1960.

[26] I. Vardi, Dedekind sums have a limiting distribution, Duke Math. J. 69, Int. Math. Research Notices (1993), 1-12.

[27] D. V. Widder, The Laplace Transform, Princeton University Press, 1946.

MATHEMATISCH INSTITUUT

UNIVERSITEIT UTRECHT

POSTBUS 80.010

3508 TA UTRECHT, THE NETHERLANDS

E-mail: BRUGGEMAN@MATH.RUU.NL 